\title{
MUON COLLIDER DESIGN
}

\author{
R. Palmer ${ }^{1,2}$, A. Sessler ${ }^{3}$, A. Skrinsky ${ }^{4}$, A. Tollestrup ${ }^{5}$, \\ A. Baltz ${ }^{1}$, S. Caspi ${ }^{3}$, P. Chen ${ }^{2}$, W-H. Cheng ${ }^{3}$, Y. Cho ${ }^{6}$, \\ D. Cline ${ }^{7}$, E. Courant ${ }^{1}$, R. Fernow ${ }^{1}$, J. Gallardo ${ }^{1}$, A. Garren ${ }^{3,7}$, \\ H. Gordon ${ }^{1}$, M. Green ${ }^{3}$, R. Gupta ${ }^{1}$, A. Hershcovitch ${ }^{1}$, \\ C. Johnstone ${ }^{5}$, S. Kahn ${ }^{1}$, H. Kirk ${ }^{1}$, T. Kycia ${ }^{1}$, Y. Lee ${ }^{1}$, \\ D. Lissauer ${ }^{1}$, A. Luccio ${ }^{1}$, A. McInturff ${ }^{3}$, F. Mills ${ }^{5}$, N. Mokhov ${ }^{5}$, \\ G. Morgan ${ }^{1}$, D. Neuffer ${ }^{5}, \mathrm{~K}-\mathrm{Y} . \mathrm{Ng}^{5}$, R. Noble $^{5}$, J. Norem ${ }^{6}$, \\ B. Norum ${ }^{8}$, K. Oide ${ }^{9}$, Z. Parsa ${ }^{1}$, V. Polychronakos ${ }^{1}$, \\ M. Popovic ${ }^{5}$, P. Rehak ${ }^{1}$, T. Roser ${ }^{1}$, R. Rossmanith ${ }^{10}$, \\ R. Scanlan ${ }^{3}$, L. Schachinger ${ }^{3}$, G. Silvestrov ${ }^{4}$, I. Stumer ${ }^{1}$, \\ D. Summers ${ }^{11}$, M. Syphers ${ }^{1}$, H. Takahashi ${ }^{1}$, Y. Torun ${ }^{1,12}$, \\ D. Trbojevic ${ }^{1}$, W. Turner ${ }^{3}$, A. Van Ginneken ${ }^{5}$, \\ T. Vsevolozhskaya ${ }^{4}$, R. Weggel ${ }^{13}$, E. Willen ${ }^{1}$, W. Willis ${ }^{1,14}$, \\ D. Winn ${ }^{15}$, J. Wurtele ${ }^{16}$, Y. Zhao ${ }^{1}$
}

1) Brookhaven National Laboratory, Upton, NY 11973-5000, USA

2) Stanford Linear Accelerator Center, Stanford, CA 94309, USA

3) Lawrence Berkeley National Laboratory, Berkeley, CA 94720, USA

4) BINP, RU-630090 Novosibirsk, Russia

5) Fermi National Accelerator Laboratory, Batavia, IL 60510, USA

6) Argonne National Laboratory, Argonne, IL 60439-4815, USA

7) Center for Advanced Accelerators, UCLA, Los Angeles, CA 90024-1547,USA

8) University of Virginia, Charlottesville, VA 22901, USA

9) KEK, Tsukuba-shi, Ibaraki-Ken 305, Japan

10) DESY, Hamburg, Germany

11) University of Mississippi, Oxford, MS 38677, USA

12) SUNY, Stony Brook, NY 11974, USA

13) Francis Bitter National Magnet Laboratory, MIT, Cambridge, MA 02139, USA

14) Columbia University, New York, NY 10027, USA

15) Fairfield University, Fairfield, CT 06430-5195, USA

16) UC Berkeley, Berkeley, CA 94720-7300, USA 


\begin{abstract}
Muon Colliders have unique technical and physics advantages and disadvantages when compared with both hadron and electron machines. They should thus be regarded as complementary. Parameters are given of $4 \mathrm{TeV}$ and $0.5 \mathrm{TeV}$ high luminosity $\mu^{+} \mu^{-}$colliders, and of a $0.5 \mathrm{TeV}$ lower luminosity demonstration machine. We discuss the various systems in such muon colliders, starting from the proton accelerator needed to generate the muons and proceeding through muon cooling, acceleration and storage in a collider ring. Detector background, polarization, and nonstandard operating conditions are discussed.
\end{abstract}

\title{
1 INTRODUCTION
}

\subsection{Technical Considerations}

The possibility of muon colliders was introduced by Skrinsky et al. [1] and Neuffer [2]. More recently, several workshops and collaboration meetings have greatly increased the level of discussion [3], [4]. In this paper we present scenarios for $4 \mathrm{TeV}$ and $0.5 \mathrm{TeV}$ colliders based on an optimally designed proton source, and for a lower luminosity $0.5 \mathrm{TeV}$ demonstration based on an upgraded version of the AGS. It is assumed that a demonstration version based on upgrades of the FERMILAB machines would also be possible (see second Ref. [4]).

Hadron collider energies are limited by their size, and technical constraints on bending magnetic fields. At very high energies it will also become impractical to obtain the required luminosities, which must rise as the energy squared. $e^{+} e^{-}$colliders, because they undergo simple, single-particle interactions, can reach higher energy final states than an equivalent hadron machine. However, extension of $e^{+} e^{-}$colliders to multi-TeV energies is severely performanceconstrained by beamstrahlung, and cost-constrained because two full energy linacs are required [6] to avoid the excessive synchrotron radiation that would occur in rings. Muons $\left(\frac{m_{\mu}}{m_{e}}=207\right)$ have the same advantage in energy reach as electrons, but have negligible beamstrahlung, and can be accelerated and stored in rings, making the possibility of high energy $\mu^{+} \mu^{-}$colliders attractive. There are however, several major technical problems with $\mu$ 's:

- they decay with a lifetime of $2.2 \times 10^{-6} \mathrm{~s}$. This problem is partially overcome by rapidly increasing the energy of the muons, and thus benefiting from their relativistic $\gamma$ factor. At $2 \mathrm{TeV}$, for example, their lifetime is $0.044 \mathrm{~s}$ : sufficient for approximately 1000 storage-ring collisions;

- another consequence of the muon decays is that the decay products heat the magnets of the collider ring and create backgrounds in the detector;

- Since the muons are created through pion decay into a diffuse phase space, some form of cooling is essential. Conventional stochastic or synchrotron cooling is too slow to be effective before they decay. Ionization cooling, 
can be used, but the final emittance of the muon beams will remain larger than that possible for electrons in an $e^{+} e^{-}$collider.

Despite these problems it appears possible that high energy muon colliders might have luminosities comparable to or, at energies of several $\mathrm{TeV}$, even higher than those in $e^{+} e^{-}$colliders [5]. And because the $\mu^{+} \mu^{-}$machines would be much smaller [7], and require much lower precision (the final spots are about three orders of magnitude larger), they may be significantly less expensive. It must be remembered, however, that a $\mu^{+} \mu^{-}$collider remains a new and untried concept, and its study has just begun; it cannot yet be compared with the more mature designs for an $e^{+} e^{-}$collider.

\subsection{Physics Considerations}

There are at least two physics advantages of a $\mu^{+} \mu^{-}$collider, when compared with an $e^{+} e^{-}$collider:

- Because of the lack of beamstrahlung, a $\mu^{+} \mu^{-}$collider can be operated with an energy spread of as little as $0.01 \%$. It is thus possible to use the $\mu^{+} \mu^{-}$collider for precision measurements of masses and widths, that would be hard, if not impossible, with an $e^{+} e^{-}$collider.

- The direct coupling of a lepton-lepton system to a Higgs boson has a cross section that is proportional to the square of the mass of the lepton. As a result, the cross section for direct Higgs production from the $\mu^{+} \mu^{-}$system is 40,000 times that from an $e^{+} e^{-}$system.

However, there are liabilities:

- It will be relatively hard to obtain both high polarization and good luminosity in a $\mu^{+} \mu^{-}$collider, whereas good polarization of one beam can be obtained in an $e^{+} e^{-}$collider without any loss in luminosity. One notes however that in the muon case, moderate polarization could be obtained for both beams.

- because of the decays of the muons, there will be a considerable background of photons, muons and neutrons in the detector. This background may be acceptable for some experiments, but it cannot be as clean as in an $e^{+} e^{-}$collider.

\subsection{Discussion}

We conclude that a muon collider has both technical advantages and disadvantages when compared with an $e^{+} e^{-}$machine. Similarly it has specific physics advantages and disadvantages. It thus seems reasonable to consider

$\mu^{+} \mu^{-}$colliders as complementary to $e^{+} e^{-}$colliders, just as $e^{+} e^{-}$colliders are complementary to hadron machines. 


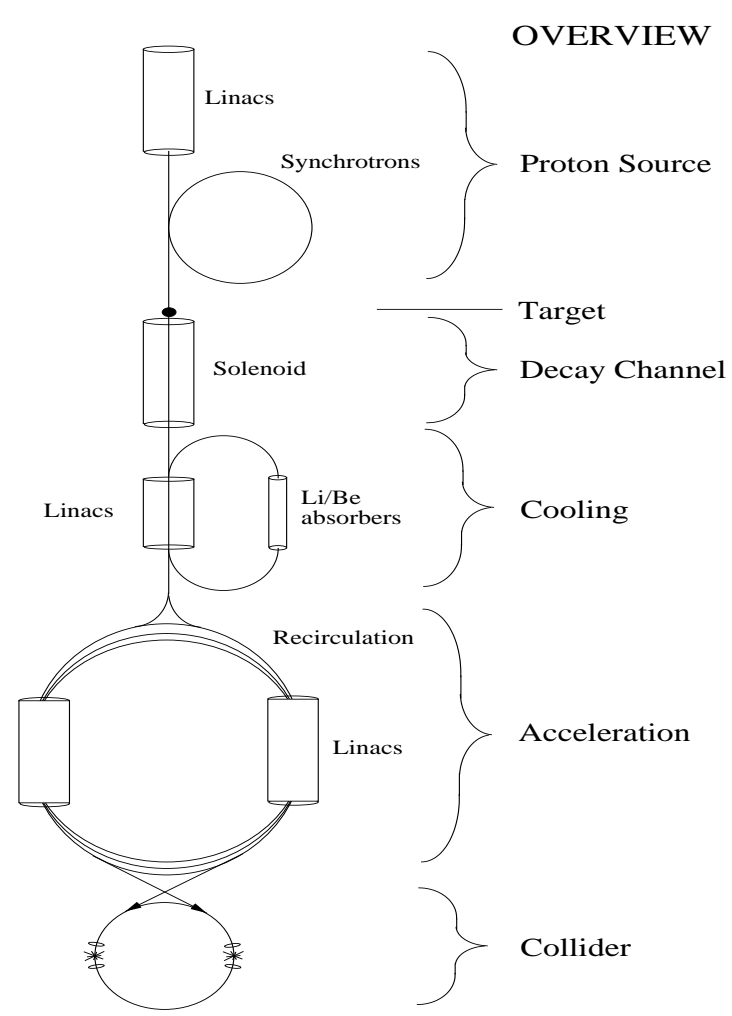

FIGURE 1. Schematic of a Muon Collider.

\subsection{Overview of Components}

The basic components of the $\mu^{+} \mu^{-}$collider are shown schematically in Fig.1. Tb. 1 shows parameters for the candidate designs. The normalized emittance $\epsilon^{N}$ is defined as the rms transverse phase space divided by $\pi$. Notice that more precisely a factor of $\pi$ must appear in the dimensions of emittance (i.e. $\pi \mathrm{mm}$ mrad). A high intensity proton source is bunch compressed and focussed on a heavy metal target. The pions generated are captured by a high field solenoid and transferred to a solenoidal decay channel within a low frequency linac. The linac serves to reduce, by phase rotation, the momentum spread of the pions, and of the muons into which they decay. Subsequently, the muons are cooled by a sequence of ionization cooling stages. Each stage consists of energy loss, acceleration, and emittance exchange by energy absorbing wedges in the presence of dispersion. Once they are cooled the muons must be rapidly accelerated to avoid decay. This can be done in recirculating accelerators (à la CEBAF) or in fast pulsed synchrotrons. Collisions occur in a separate high field collider storage ring with a single very low beta insertion. 


\begin{tabular}{|c|c|c|c|c|}
\hline & & $4 \mathrm{TeV}$ & $.5 \mathrm{TeV}$ & Demo. \\
\hline Beam energy & $\mathrm{TeV}$ & 2 & .25 & .25 \\
\hline Beam $\gamma$ & & 19,000 & 2,400 & 2,400 \\
\hline Repetition rate & $\mathrm{Hz}$ & 15 & 15 & 2.5 \\
\hline Muons per bunch & $10^{12}$ & 2 & 4 & 4 \\
\hline Bunches of each sign & & 2 & 1 & 1 \\
\hline Normalized rms emittance $\epsilon^{N}$ & $\pi \mathrm{mm} \operatorname{mrad}$ & 50 & 90 & 90 \\
\hline Bending Field & $\mathrm{T}$ & 9 & 9 & 8 \\
\hline Circumference & $\mathrm{Km}$ & 7 & 1.2 & 1.5 \\
\hline Average ring mag. field $B$ & $\mathrm{~T}$ & 6 & 5 & 4 \\
\hline Effective turns before decay & & 900 & 800 & 750 \\
\hline$\beta^{*}$ at intersection & $\mathrm{mm}$ & 3 & 8 & 8 \\
\hline rms beam size at I.P. & $\mu m$ & 2.8 & 17 & 17 \\
\hline Luminosity & $\mathrm{cm}^{-2} \mathrm{~s}^{-1}$ & $10^{35}$ & $510^{33}$ & $610^{32}$ \\
\hline
\end{tabular}

TABLE 1. Parameters of Collider Rings

\section{MUON PRODUCTION}

\subsection{Proton Driver}

The specifications of the proton drivers are given in Tb.2. In the examples, it is a high-intensity $\left(2.5 \times 10^{13}\right.$ protons per pulse $) 30 \mathrm{GeV}$ proton synchrotron. The preferred cycling rate would be $15 \mathrm{~Hz}$, but for a demonstration machine using the AGS [8], the repetition rate would be limited to $2.5 \mathrm{~Hz}$ and to $24 \mathrm{GeV}$. For the lower energy machines, 2 final bunches are employed (one to make $\mu^{-}$'s and the other to make $\mu^{+}$'s). For the high energy collider, four are used (two $\mu$ bunches of each sign).

Earlier studies had suggested that the driver could be a $10 \mathrm{GeV}$ machine with the same charge per bunch, but a repetition rate of $30 \mathrm{~Hz}$. This specification was almost identical to that studied [9] at ANL for a spallation neutron source. Studies at FNAL [10] have further established that such a specification is reasonable. But in order to reduce the cost of the muon phase rotation section and for minimizing the final muon longitudinal phase space, it appears now that the final proton bunch length should be $1 \mathrm{~ns}$ (or even less). This appears difficult to achieve at $10 \mathrm{GeV}$, but possible at $30 \mathrm{GeV}$.

\begin{tabular}{|c|c|c|c|c|}
\hline & & $4 \mathrm{TeV}$ & $5 \mathrm{TeV}$ & Demo \\
\hline Proton energy & $\mathrm{GeV}$ & 30 & 30 & 24 \\
\hline Repetition rate & $\mathrm{Hz}$ & 15 & 15 & 2.5 \\
\hline Protons per bunch & $10^{13}$ & 2.5 & 2.5 & 2.5 \\
\hline Bunches & & 4 & 2 & 2 \\
\hline Long. phase space/bunch & $\mathrm{eV} \mathrm{s}$ & 4.5 & 4.5 & 4.5 \\
\hline Final $r m s$ bunch length & ns & 1 & 1 & 1 \\
\hline
\end{tabular}

TABLE 2. Proton Driver Specifications 


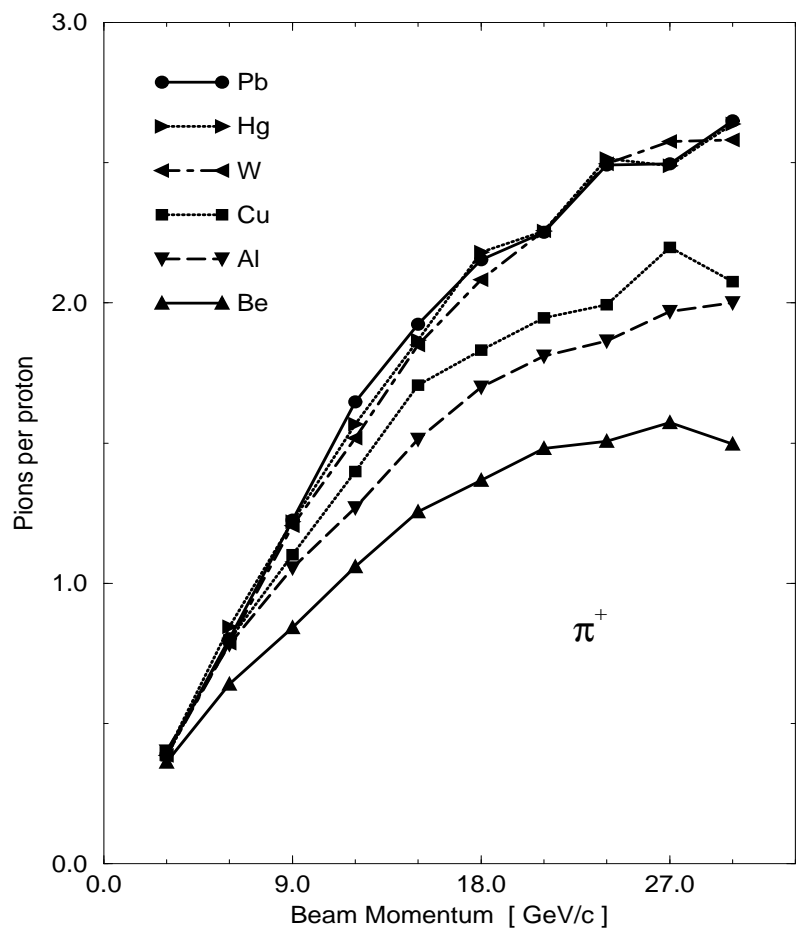

FIGURE 2. ARC forward $\pi^{+}$production vs proton energy and target material.

A $1 \mathrm{~ns}$ rms bunch at $30 \mathrm{GeV}$ with a phase space per bunch of $6 \pi \sigma_{t} \sigma_{E}=$ $4.5 \mathrm{eVs}$ (at 95\%) bunch, will have a momentum spread of $0.8 \%,(2 \%$ at $95 \%)$, and the space charge tune shift just before extraction would be $\approx 0.5$. Provided the rotation can be performed rapidly enough, this should not be a problem.

An attractive technique [11] for bunch compression would be to generate a large momentum spread with modest rf at a final energy close to transition. Pulsed quads would then be employed to move the operating point away from transition, resulting in rapid compression.

\subsection{Target and Pion Capture}

Predictions of the nuclear Monte-Carlo program ARC [12] suggest that $\pi$ production is maximized by the use of heavy target materials, and that the production is peaked at a relatively low pion energy $(\approx 100 \mathrm{MeV})$, substantially independent of the initial proton energy. Fig.2 shows the forward $\pi^{+}$ production as a function of proton energy and target material; the $\pi^{-}$distributions are similar. Other programs [13], [14] do not predict such a large low energy peak, and there is currently very little data to indicate which is right. An experiment (E910), currently running at the AGS, should decide this question, and thus settle at which energy the capture should be optimized. 


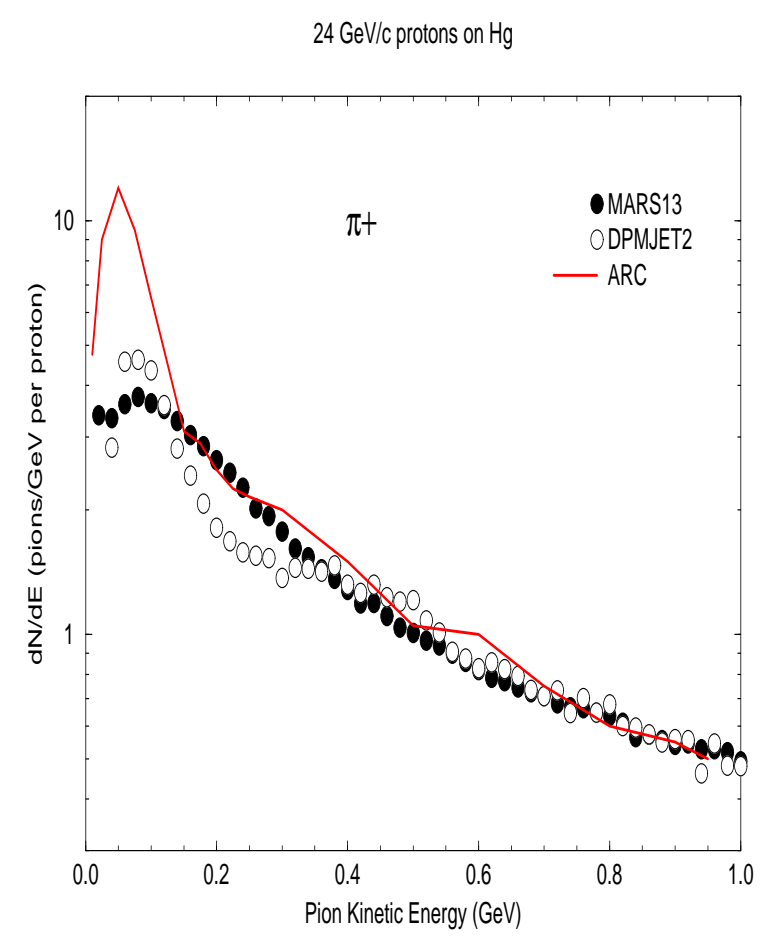

FIGURE 3. $\pi^{+}$energy distribution for $24 \mathrm{GeV}$ protons on $\mathrm{Hg}$.

The target would probably be made of $\mathrm{Cu}$, approximately $24 \mathrm{~cm}$ long by 2 $\mathrm{cm}$ diameter. A study [15] indicates that, with a $3 \mathrm{~mm}$ rms beam, the single pulse instantaneous temperature rise is acceptable, but, if cooling is only supplied from the outside, the equilibrium temperature would be excessive. Some method must be provided to give cooling within the target volume. For instance, the target could be made of a stack of relatively thin copper disks, with water cooling between them.

Figs.3,4 compared the predictions of the mentioned codes, for the energy distribution of $\pi^{+}$and $\pi^{-}$for $24 \mathrm{GeV}$ protons on $\mathrm{Hg}$; the distributions for $\mathrm{Cu}$ are similar.

Pions are captured from the target by a high-field $(20 \mathrm{~T}, 15 \mathrm{~cm}$ inside diameter) hybrid magnet: superconducting on the outside, and a water cooled Bitter solenoid on the inside. A preliminary design [16] (see Fig.5) has an inner Bitter magnet with an inside diameter of $24 \mathrm{~cm}$ (space is allowed for a $4 \mathrm{~cm}$ heavy metal shield inside the coil) and an outside diameter of $60 \mathrm{~cm}$; it provides half $(10 \mathrm{~T})$ of the total field, and would consume approximately $8 \mathrm{MW}$. The superconducting magnet has a set of three coils, all with inside diameters of $70 \mathrm{~cm}$ and is designed to give $10 \mathrm{~T}$ at the target and provide the required tapered field [17] (see Fig.6) to match into the periodic superconducting solenoidal decay channel $(5 \mathrm{~T}$ and radius $=15 \mathrm{~cm})$. 


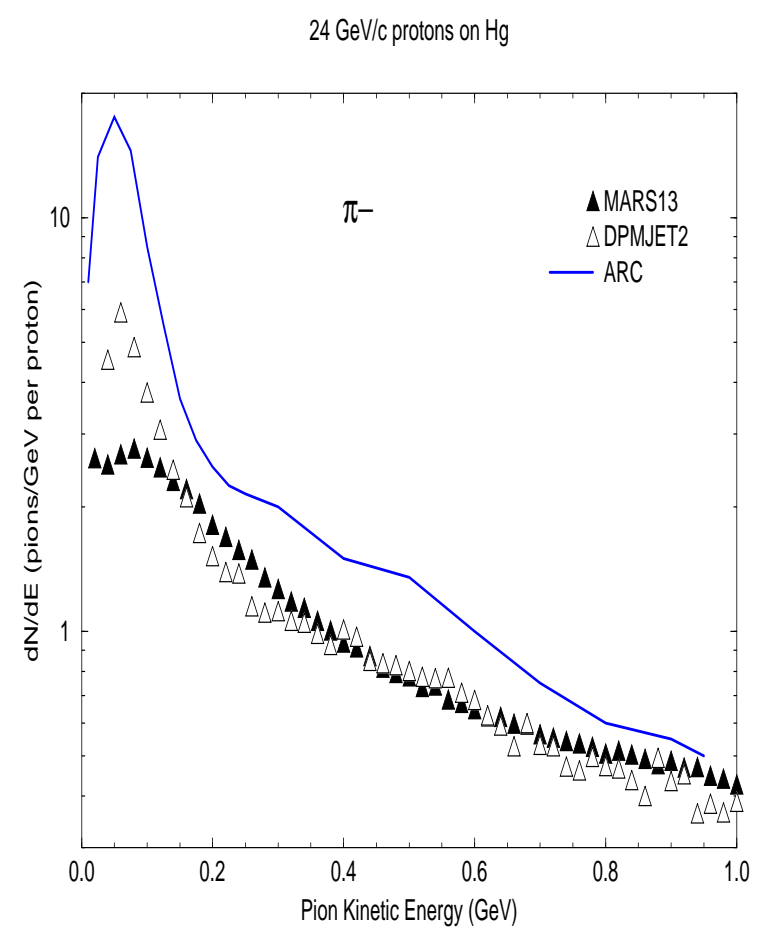

FIGURE 4. $\pi^{-}$energy distribution for $24 \mathrm{GeV}$ protons on $\mathrm{Hg}$.

Monte Carlo studies indicate a yield of 0.4-0.6 muons, of each sign, per initial proton, captured in the decay channel. Surprisingly, this conclusion seems relatively independent of whether the system is optimised for energies of 50 to $500 \mathrm{MeV}$ (using ARC), or 200 to $2000 \mathrm{MeV}$ (using MARS).

\subsection{Use of Both Signs}

Protons on the target produce pions of both signs, and a solenoid will capture both, but the required subsequent phase rotation rf systems will have opposite effects on each. One solution is to break the proton bunch into two, aim them on the same target one after the other, and adjust the rf phases such as to act correctly on one sign of the first bunch and on the other sign of the second. This is the solution assumed in the parameters of this paper.

A second possibility would be to: 1) to separate the charges into two channels, 2) delay the particles of one charge by introducing a chikane in one of the channels, 3) recombine the two channels so that the particles of the two charges are in line, but separated longitudinally (i.e. box cared). Both charges can now be phase rotated by a single linac with appropriate phases of rf.

A third solution is to separate the pions of each charge prior to the use of rf, and feed the beams of each charge into different channels. 
After the target, and prior to the use of any rf or cooling, the beams have very large emittances and energy spread. Conventional charge separation using a dipole is not practical. But if a solenoidal channel is bent, then the particles trapped within that channel will drift [18] in a direction perpendicular to the bend. With our parameters this drift is dominated by a term (curvature drift) that is linear with the forward momentum of the particles, and has a direction that depends on the sign of the charges. If sufficient bend is employed [15], the two charges could then be separated by a septum and captured into two separate channels. When these separate channels are bent back to the same forward direction, the momentum dispersion is separately removed in each new channel.

Although this idea is very attractive, it has some problems:

- If the initial beam has a radius $\mathrm{r}=0.15 \mathrm{~m}$, and if the momentum range to be accepted is $F=\frac{p_{\max }}{p_{\min }}=3$, then the required height of the solenoid just prior to separation is $2(1+F) \mathrm{r}=1.2 \mathrm{~m}$. Use of a lesser height will result in particle loss. Typically, the reduction in yield for a curved solenoid compared to a straight solenoid is about $25 \%$ (due to the loss of very low and very high momentum pions), but this must be weighed against the fact that both charge signs are captured for each proton on target.
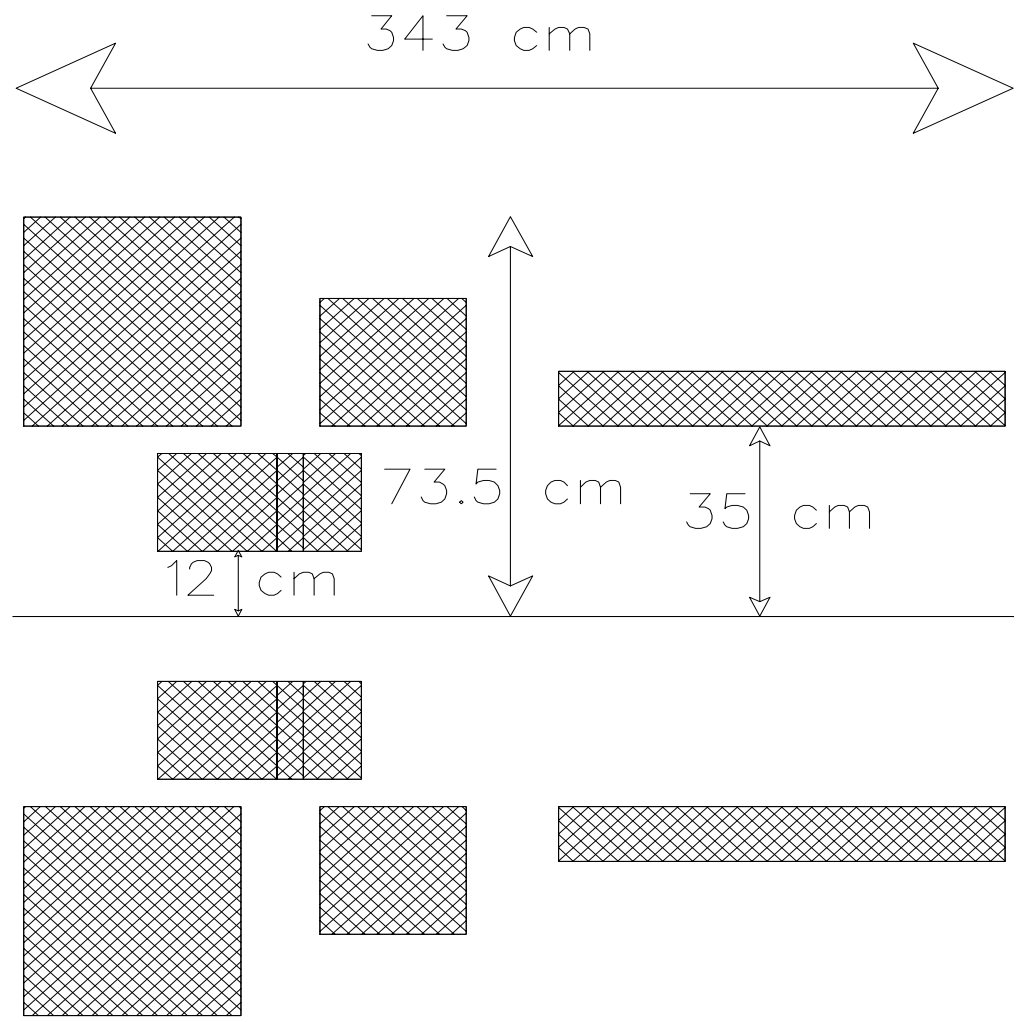

FIGURE 5. Schematic of a hybrid magnet solenoid system for $\pi$ capture and matching. 
- The system of bend, separate, and return bend will require significant length and must occur prior to the start of phase rotation (see below). Unfortunately, it appears that the cost of the phase rotation rf is strongly dependent on keeping this distance as short as possible. On the other hand a bent solenoid would separate the remnant proton beam and other charged debris exiting the target before the rf cavities.

Clearly, compromises will be involved, and more study of this concept is required.

\subsection{Phase Rotation Linac}

The pions, and the muons into which they decay, have an energy spread from about $0-3 \mathrm{GeV}$, with an $\mathrm{rms} /$ mean of $\approx 100 \%$, and with a peak at about $100 \mathrm{MeV}$. It would be difficult to handle such a wide spread in any subsequent system. A linac is thus introduced along the decay channel, with frequencies and phases chosen to deaccelerate the fast particles and accelerate the slow ones; i.e. to phase rotate the muon bunch. Tb.3 gives an example of parameters of such a linac. It is seen that the lowest frequency is $30 \mathrm{MHz}$, a low but not impossible frequency for a conventional structure.

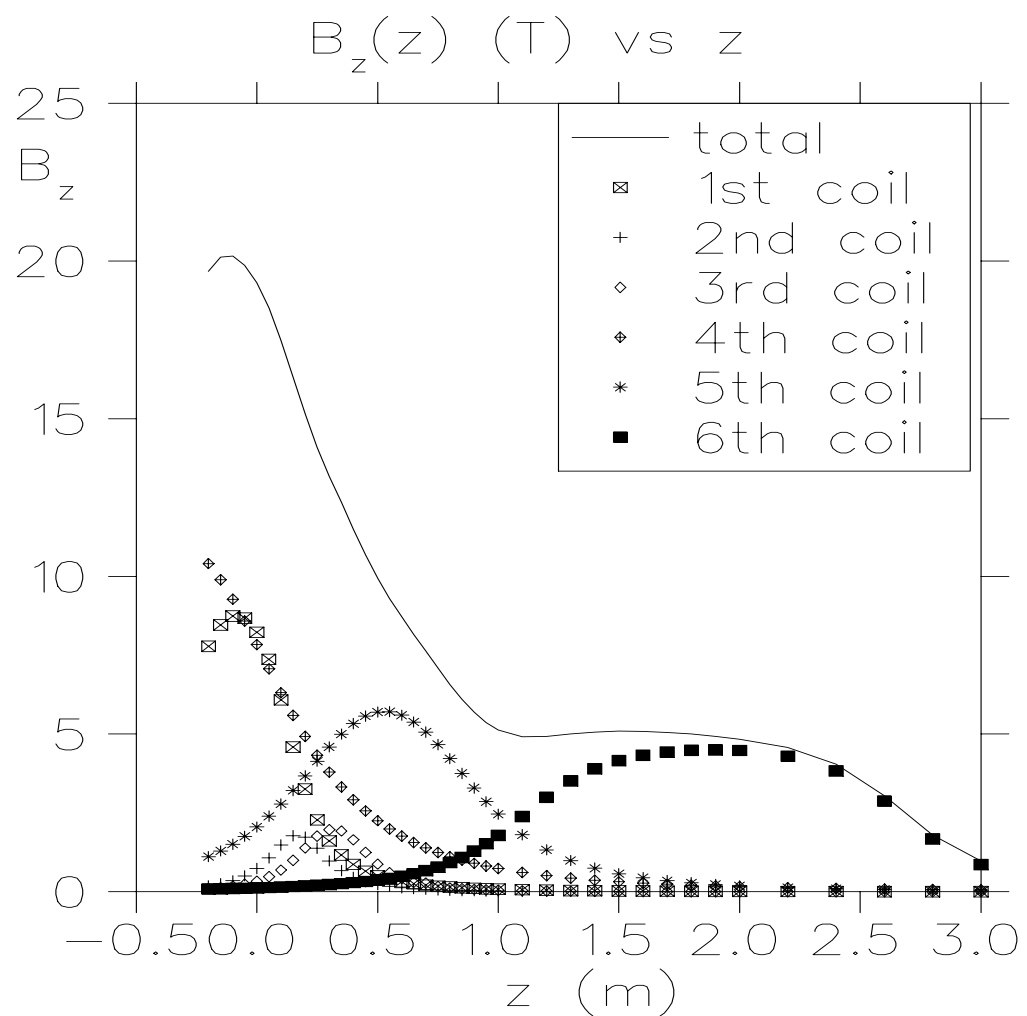

FIGURE 6. Total and individual component field profiles of hybrid magnet solenoid. 


\begin{tabular}{cccc}
\hline \multirow{4}{*}{$\begin{array}{c}\text { Linac } \\
\end{array}$} & \multicolumn{3}{c}{ Length } \\
$\mathrm{m}$ & $\begin{array}{c}\text { Frequency } \\
\text { MHz }\end{array}$ & $\begin{array}{c}\text { Gradient } \\
\mathrm{MeV} / \mathrm{m}\end{array}$ \\
\hline 1 & 3 & 60 & 5 \\
2 & 29 & 30 & 4 \\
3 & 5 & 60 & 4 \\
4 & 5 & 37 & 4 \\
\hline
\end{tabular}

TABLE 3. Parameters of Phase Rotation Linacs

A design of a reentrant $30 \mathrm{MHz}$ cavity is shown in Fig.7. Its parameters are given in Tb.4. It has a diameter of approximately $2 \mathrm{~m}$, only about one

\begin{tabular}{llc}
\hline Cavity Radius & $\mathrm{cm}$ & 101 \\
Cavity Length & $\mathrm{cm}$ & 120 \\
Beam Pipe Radius & $\mathrm{cm}$ & 15 \\
Accelerating Gap & $\mathrm{cm}$ & 24 \\
Q & 18200 \\
Average Acceleration Gradient $\mathrm{MV} / \mathrm{m}$ & 3 \\
Peak rf Power & $\mathrm{MW}$ & 6.3 \\
Average Power $(15 \mathrm{~Hz})$ & $\mathrm{KW}$ & 18.2 \\
Stored Energy & $\mathrm{J}$ & 609 \\
\hline
\end{tabular}

TABLE 4. Parameters of $30 \mathrm{MHz}$ rf Cavity

third of that of a conventional pill-box cavity. To keep its cost down, it would be made of Al. Multipactoring would probably be suppressed by stray fields from the $5 \mathrm{~T}$ focusing coils, but could also be controlled by an internal coating of titanium nitride.

Figs. 8 and 9 show the energy vs ct at the end of the decay channel with and without phase rotation. Note that the $\mathrm{ct}$ scales are very different: the rotation both compacts the energy spread and limits the growth of the bunch length.

After this phase rotation, a bunch can be selected with mean energy 150 $\mathrm{MeV}$, rms bunch length $1.7 \mathrm{~m}$, and rms momentum spread $20 \%\left(95 \%, \epsilon_{\mathrm{L}}=\right.$ $3.2 \mathrm{eVs}$ ). The number of muons per initial proton in this selected bunch is $0.2-0.3$, about half the total number of pions initially captured. As noted above, since the linacs cannot phase rotate both signs in the same bunch, we need two bunches: the phases are set to rotate the $\mu^{+}$'s of one bunch and the $\mu^{-}$'s of the other. Prior to cooling, the bunch is accelerated to $300 \mathrm{MeV}$, in order to reduce the momentum spread to $10 \%$.

\section{COOLING}

For collider intensities, the phase-space volume must be reduced within the $\mu$ lifetime. Cooling by synchrotron radiation, conventional stochastic cooling 
FIGURE 7. $30 \mathrm{MHz}$ cavity for use in phase rotation and early stages of cooling.

and conventional electron cooling are all too slow. Optical stochastic cooling [19], electron cooling in a plasma discharge [20] and cooling in a crystal lattice [21] are being studied, but appear very difficult. Ionization cooling [22] of muons seems relatively straightforward.

\subsection{Ionization Cooling Theory}

In ionization cooling, the beam loses both transverse and longitudinal momentum as it passes through a material medium. Subsequently, the longitudinal momentum can be restored by coherent reacceleration, leaving a net loss of transverse momentum. Ionization cooling is not practical for protons and electrons because of nuclear interactions (p's) and bremsstrahlung (e's), but is practical for $\mu$ 's because of their low nuclear cross section and relatively low bremsstrahlung.

The equation for transverse cooling (with energies in $\mathrm{GeV}$ ) is:

$$
\frac{d \epsilon_{n}}{d s}=-\frac{d E_{\mu}}{d s} \frac{\epsilon_{n}}{E_{\mu}}+\frac{\beta_{\perp}(0.014)^{2}}{2 E_{\mu} m_{\mu} L_{R}}
$$

where $\epsilon_{n}$ is the normalized emittance, $\beta_{\perp}$ is the betatron function at the absorber, $d E_{\mu} / d s$ is the energy loss, and $L_{R}$ is the radiation length of the material. The first term in this equation is the coherent cooling term, and 


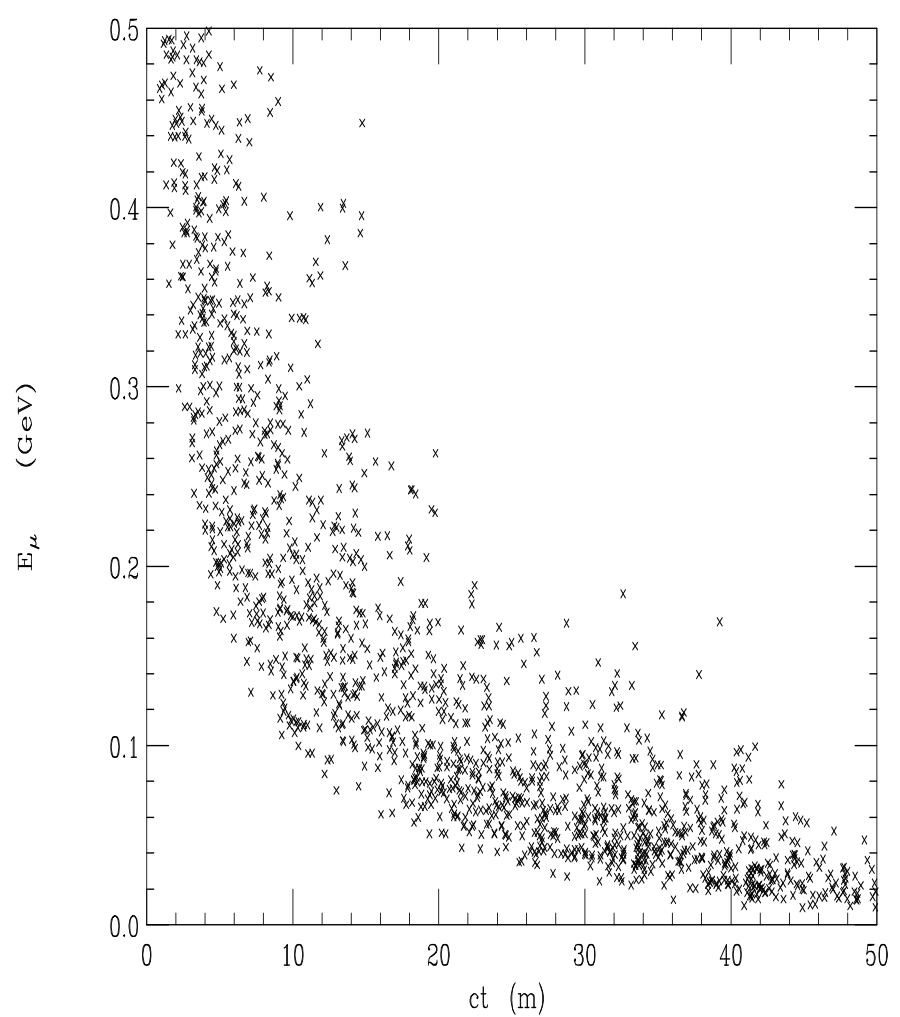

FIGURE 8. Energy vs ct of Muons at End of Decay Channel without Phase Rotation.

the second is the heating due to multiple scattering. This heating term is minimized if $\beta_{\perp}$ is small (strong-focusing) and $L_{R}$ is large (a low-Z absorber). From Eq.1 we find a limit to transverse cooling, which occurs when heating due to multiple scattering balances cooling due to energy loss. The limits are $\epsilon_{n} \approx 0.610^{-2} \beta_{\perp}$ for $\mathrm{Li}$, and $\epsilon_{n} \approx 0.810^{-2} \beta_{\perp}$ for Be.

The equation for energy spread (longitudinal emittance) is:

$$
\frac{d(\Delta E)^{2}}{d s}=-2 \frac{d\left(\frac{d E_{\mu}}{d s}\right)}{d E_{\mu}}<\left(\Delta E_{\mu}\right)^{2}>+\frac{d\left(\Delta E_{\mu}\right)_{\text {straggling }}^{2}}{d s}
$$

where the first term is the cooling (or heating) due to energy loss, and the second term is the heating due to straggling.

Cooling requires that $\frac{d\left(d E_{\mu} / d s\right)}{d E_{\mu}}>0$. But at energies below about $200 \mathrm{MeV}$, the energy loss function for muons, $d E_{\mu} / d s$, is decreasing with energy and there is thus heating of the beam. Above $400 \mathrm{MeV}$ the energy loss function increases gently, giving some cooling, but not sufficient for our application.

Energy spread can also be reduced by artificially increasing $\frac{d\left(d E_{\mu} / d s\right)}{d E_{\mu}}$ by placing a transverse variation in absorber density or thickness at a location where position is energy dependent, i.e. where there is dispersion. The use of such wedges can reduce energy spread, but it simultaneously increases transverse 


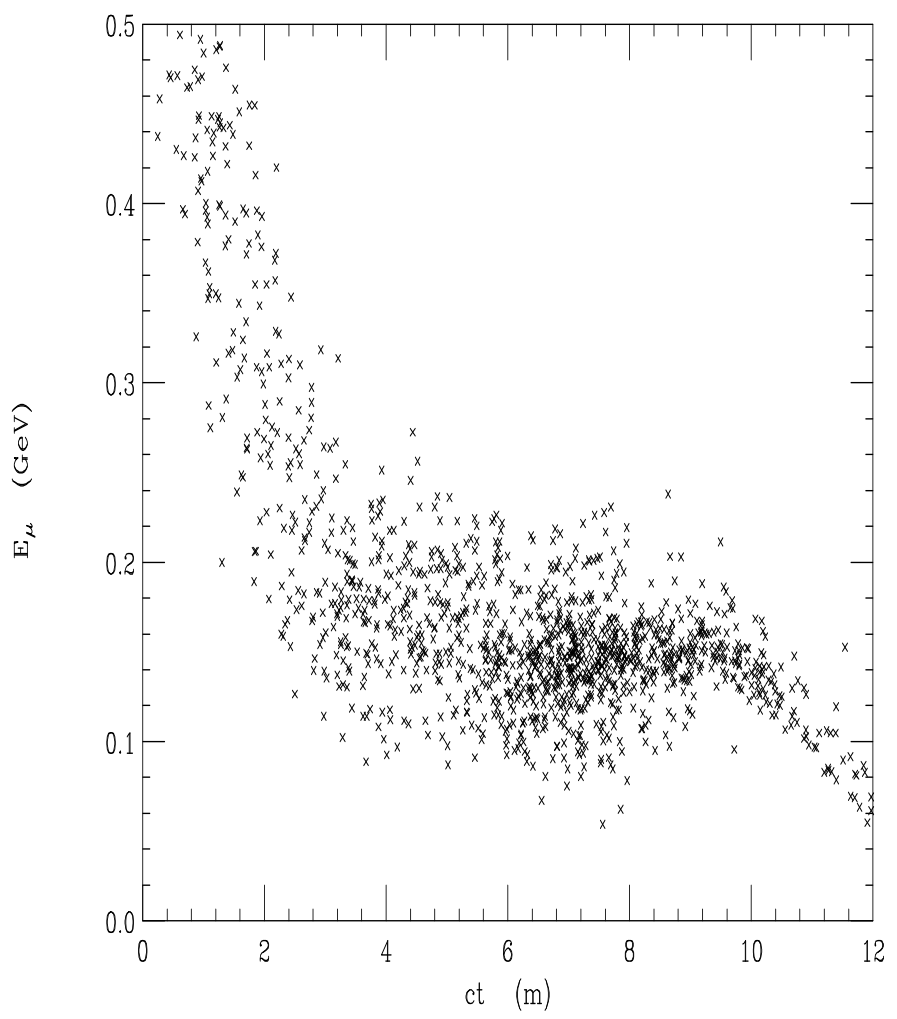

FIGURE 9. Energy vs ct of Muons at End of Decay Channel with Phase Rotation.

emittance in the direction of the dispersion. Six dimensional phase space is not reduced, but it does allow the exchange of emittance between the longitudinal and transverse directions.

In the long-path-length Gaussian-distribution limit, the heating term (energy straggling) is given by [23]

$$
\frac{d\left(\Delta E_{\mu}\right)_{\text {straggling }}^{2}}{d s}=4 \pi\left(r_{e} m_{e} c^{2}\right)^{2} N_{o} \frac{Z}{A} \rho \gamma^{2}\left(1-\frac{\beta^{2}}{2}\right),
$$

where $N_{o}$ is Avogadro's number and $\rho$ is the density. Since the energy straggling increases as $\gamma^{2}$, and the cooling system size scales as $\gamma$, cooling at low energies is desired.

\subsection{Cooling System}

We require a reduction of the normalized transverse emittance by almost three orders of magnitude (from $1 \times 10^{-2}$ to $5 \times 10^{-5} \mathrm{~m}-\mathrm{rad}$ ), and a reduction of the longitudinal emittance by one order of magnitude. This cooling is obtained in a series of cooling stages. In general, each stage consists of three components with matching sections between them: 
1. a FOFO lattice consisting of spaced axial solenoids with alternating field directions and lithium hydride absorbers placed at the centers of the spaces between them, where the $\beta_{\perp}$ 's are minimum.

2. a lattice consisting of more widely separated alternating solenoids, and bending magnets between them to generate dispersion. At the location of maximum dispersion, wedges of lithium hydride are introduced to interchange longitudinal and transverse emittance.

3. a linac to restore the energy lost in the absorbers.

At the end of a sequence of such cooling stages, the transverse emittance can be reduced to about $10^{-3} \mathrm{~m}-\mathrm{rad}$, still a factor of $\approx 20$ above the emittance goals of Tb.1. The longitudinal emittance, however, can be cooled to a value nearly three orders of magnitude less than is required. The additional reduction of transverse emittance can then be obtained by a reverse exchange of transverse and longitudinal phase-spaces. This is again done by the use of wedged absorbers in dispersive regions between solenoid elements.

Throughout this process appropriate momentum compaction and rf fields must be used to control the bunch, in the presence of space charge, wake field and resistive wall effects.

In a few of the later stages, current carrying lithium rods might replace item (1) above. In this case the rod serves simultaneously to maintain the low $\beta_{\perp}$, and attenuate the beam momenta. Similar lithium rods, with surface fields of $10 \mathrm{~T}$, were developed at Novosibirsk and have been used as focusing elements at FNAL and CERN [24]. It is hoped [25] that liquid lithium columns, can be used to raise the surface field to $20 \mathrm{~T}$ and improve the resultant cooling. The Li or Be lenses will permit smaller $\beta_{\perp}$ and therefore more transverse cooling with the consequence that the emittance exchange with the longitudinal would be reduced.

It would be desirable, though not necessarily practical, to economize on linac sections by forming groups of stages into recirculating loops.

\subsection{Example}

A model example has been generated that uses no lithium rods and no recirculating loops. It is assumed here that each charge is cooled in a separate channel, although it might be possible to design a system with both charges in the same channel. Individual components of the lattices have been defined, but a complete lattice has not yet been specified, and no Monte Carlo study of its performance has yet been performed. Spherical aberration due to solenoid end effects, wake fields, and second order rf effects have not yet been included.

The phase advance in each cell of the lattice is made as close to $\pi$ as possible in order to minimize the $\beta$ 's at the location of the absorber, but it is kept 
somewhat less than this value so that the phase advance per cell should never exceed $\pi$. The following effects are included: the maximum space charge transverse defocusing; a $3 \sigma$ fluctuation of momentum; a $3 \sigma$ fluctuation in amplitude.

Fig.10 shows the beta function (solid-line) and phase advance (dashed-line) through two typical cells of the cooling lattice. In the early stages, the solenoids have relatively large diameters and their fields are limited to $7 \mathrm{~T}$. In later stages the emittance has decreased, the apertures are smaller and the fields are increased to $11 \mathrm{~T}$. The maximum bending fields used are $7 \mathrm{~T}$, but most are closer to $3 \mathrm{~T}$. The emittances, transverse and longitudinal, as a

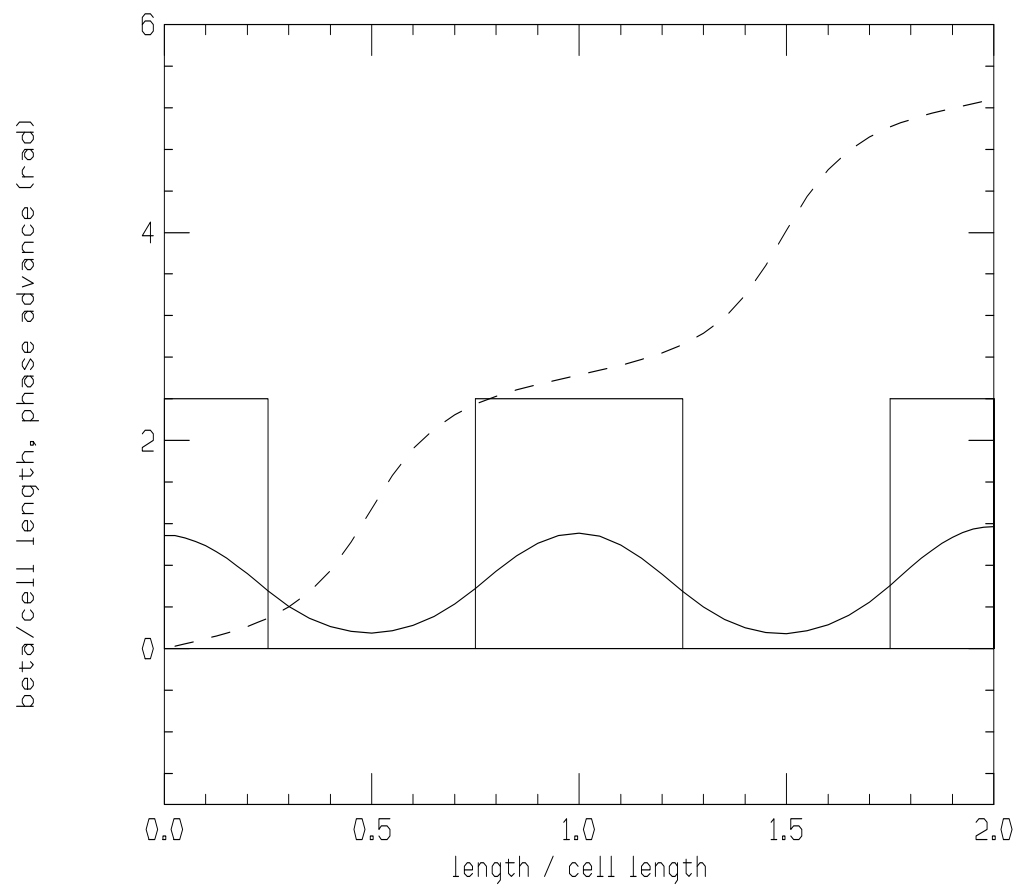

FIGURE 10. Beta function(solid) and Phase Advance(dashed) vs $z$ in FOFO cells.

function of stage number, are shown in Fig.11, together with the beam energy. In the first 15 stages, relatively strong wedges are used to rapidly reduce the longitudinal emittance, while the transverse emittance is reduced relatively slowly. The object is to reduce the bunch length, thus allowing the use of higher frequency and higher gradient $\mathrm{rf}$ in the reacceleration linacs. In the next 10 stages, the emittances are reduced close to their asymptotic limits. In the last two stages, the transverse and longitudinal emittances are again exchanged, but in the opposite direction: lowering the transverse and raising the longitudinal. During this exchange the energy is allowed to fall to $10 \mathrm{MeV}$ in order to minimize the $\beta$, and thus limit the emittance dilution.

The total length of the system is $500 \mathrm{~m}$, and the total acceleration used is $3.3 \mathrm{GeV}$. The fraction of muons remaining at the end of the cooling system is calculated to be $58 \%$. 


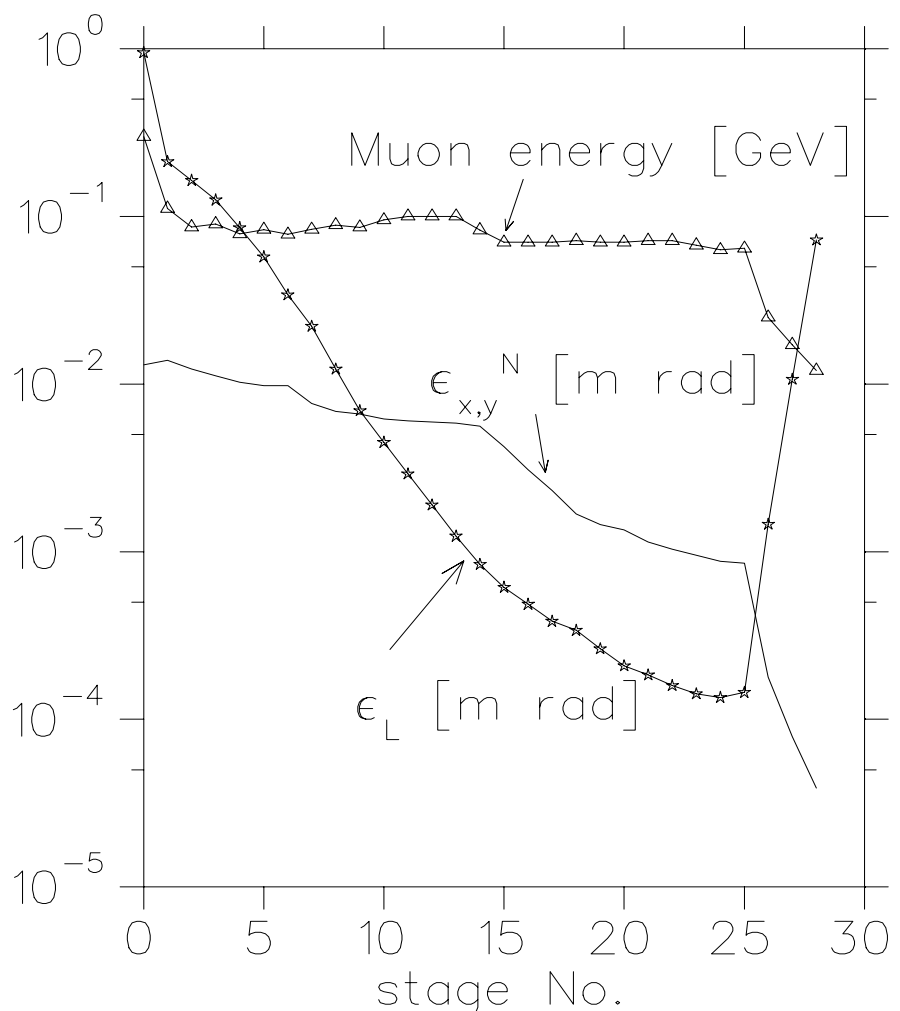

FIGURE 11. $\epsilon_{\perp}, \frac{\epsilon_{L} c}{\left\langle\mathrm{E}_{\mu}\right\rangle}$ and $\mathrm{E}_{\mu}[\mathrm{GeV}]$ vs stage number in the cooling sequence.

\section{ACCELERATION}

Following cooling and initial bunch compression the beams must be rapidly accelerated to full energy (2 TeV, or $250 \mathrm{GeV}$ ). A sequence of linacs would work, but would be expensive. Conventional synchrotrons cannot be used because the muons would decay before reaching the required energy. The conservative solution is to use a sequence of recirculating accelerators (similar to that used at CEBAF). A more economical solution would be to use fast rise time pulsed magnets in synchrotrons, or synchrotrons with rapidly rotating permanent magnets interspersed with high field fixed magnets.

\subsection{Recirculating Acceleration}

Tb.5 gives an example of a possible sequence of recirculating accelerators. After initial linacs, there are two conventional rf recirculating accelerators taking the muons up to $75 \mathrm{GeV}$, then two superconducting recirculators going up to $2000 \mathrm{GeV}$.

Criteria that must be considered in picking the parameters of such accelerators are: 


\begin{tabular}{llccccc}
\hline & & Linac & $\# 1$ & $\# 2$ & $\# 3$ & $\# 4$ \\
\hline initial energy & $\mathrm{GeV}$ & 0.20 & 1 & 8 & 75 & 250 \\
final energy & $\mathrm{GeV}$ & 1 & 8 & 75 & 250 & 2000 \\
nloop & & 1 & 12 & 18 & 18 & 18 \\
freq. & $\mathrm{MHz}$ & 100 & 100 & 400 & 1300 & 2000 \\
linac V & $\mathrm{GV}$ & 0.80 & 0.58 & 3.72 & 9.72 & 97.20 \\
grad & & 5 & 5 & 10 & 15 & 20 \\
$\mathrm{dp} / \mathrm{p}$ initial & $\%$ & 12 & 2.70 & 1.50 & 1 & 1 \\
dp/p final & $\%$ & 2.70 & 1.50 & 1 & 1 & 0.20 \\
$\sigma_{z}$ initial & $\mathrm{mm}$ & 341 & 333 & 82.52 & 14.52 & 4.79 \\
$\sigma_{z}$ final & $\mathrm{mm}$ & 303 & 75.02 & 13.20 & 4.36 & 3.00 \\
$\eta$ & $\%$ & 1.04 & 0.95 & 1.74 & 3.64 & 4.01 \\
$N_{\mu}$ & $10^{12}$ & 2.59 & 2.35 & 2.17 & 2.09 & 2 \\
$\tau_{f i l l}$ & $\mu \mathrm{S}$ & 87.17 & 87.17 & 10.90 & $\mathrm{s.c.}$ & $\mathrm{s} . \mathrm{c}$. \\
beam t & $\mu \mathrm{s}$ & 0.58 & 6.55 & 49.25 & 103 & 805 \\
decay survival & & 0.94 & 0.91 & 0.92 & 0.97 & 0.95 \\
linac len & $\mathrm{km}$ & 0.16 & 0.12 & 0.37 & 0.65 & 4.86 \\
arc len & $\mathrm{km}$ & 0.01 & 0.05 & 0.45 & 1.07 & 8.55 \\
tot circ & $\mathrm{km}$ & 0.17 & 0.16 & 0.82 & 1.72 & 13.41 \\
phase slip & $\mathrm{deg}$ & 0 & 38.37 & 7.69 & 0.50 & 0.51 \\
\hline TABLE & $\mathrm{Param}$ & & & & &
\end{tabular}

TABLE 5. Parameters of Recirculating Accelerators

- The wavelengths of rf should be chosen to limit the loading, $\eta$, (it is restricted to below $4 \%$ in this example) to avoid excessive longitudinal wakefields and the resultant emittance growth.

- The wavelength should also be sufficiently large compared to the bunch length to avoid second order effects (in this example: 10 times).

- For power efficiency, the cavity fill time should be long compared to the acceleration time. When conventional cavities cannot satisfy this condition, superconducting cavities are required.

- In order to minimize muon decay during acceleration (in this example $73 \%$ of the muons are accelerated without decay), the number of recirculations at each stage should be kept low, and the rf acceleration voltage correspondingly high. But for minimum cost, the number of recirculations appears to be of the order of 20 - a relatively high number. In order to avoid a large number of separate magnets, multiple aperture magnets can be designed (see Fig.12).

Note that the linacs see two bunches of opposite signs, passing through in opposite directions. In the final accelerator in the $2 \mathrm{TeV}$ case, each bunch passes through the linac 18 times. The total loading is then $4 \times 18 \times \eta=288 \%$. With this loading, assuming $60 \%$ klystron efficiencies and reasonable cryogenic loads, one could probably achieve $35 \%$ wall to beam power efficiency, giving a wall power consumption for the rf in this ring of $108 \mathrm{MW}$. 


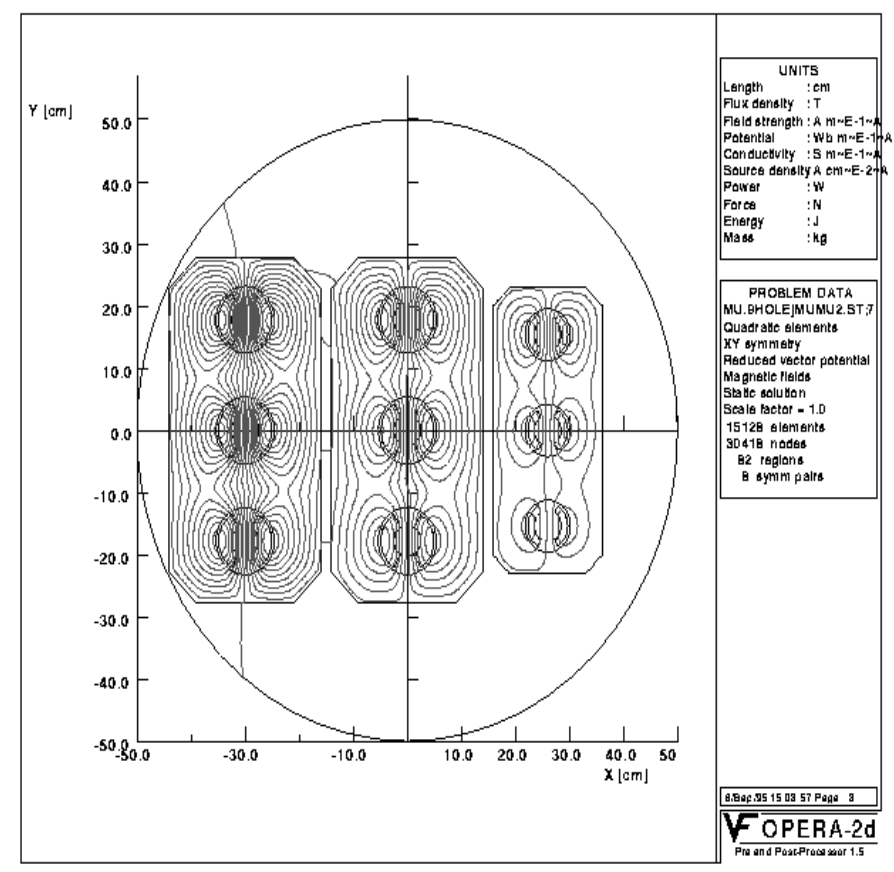

FIGURE 12. A cross section of a 9 aperture sc magnet.

A recent study [26] tracked particles through a similar sequence of recirculating accelerators and found a dilution of longitudinal phase space of the order of $10 \%$ and negligible particle loss.

\subsection{Pulsed Magnets}

An alternative to recirculating accelerators for stages \#2 and \#3 would be to use pulsed magnet synchrotrons. The cross section of a pulsed magnet for this purpose is shown in Fig.13. If desired, the number of recirculations could be higher in this case, and the needed rf voltage correspondingly lower, but the loss of particles from decay would be somewhat more. The cost for a pulsed magnet system appears to be significantly less than that of a multihole recirculating magnet system, and the power consumption is moderate for energies up to $250 \mathrm{GeV}$. Unfortunately, the power consumption is impractical at energies above about $500 \mathrm{GeV}$.

\subsection{Pulsed and Superconducting Hybrid}

For the final acceleration to $2 \mathrm{TeV}$ in the high energy machine, the power consumed by a ring using only pulsed magnets would be excessive. A recir- 


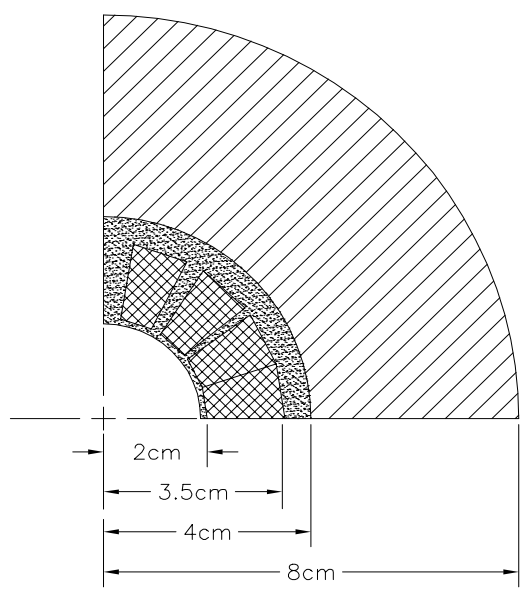

FIGURE 13. Cross section of pulsed magnet for use in the acceleration to $250 \mathrm{GeV}$.

culating accelerator is still usable, but a hybrid ring with alternating pulsed warm magnets and fixed superconducting magnets might be a cheaper alternative.

For instance: A sequence of two such hybrid accelerators could be used. One from $0.25-1 \mathrm{TeV}$, and the other from 1-2 TeV. Each would employ $10 \mathrm{~T}$ superconducting fixed magnets alternating with pulsed warm magnets whose fields would swing from $-1.5 \mathrm{~T}$ to $+1.5 \mathrm{~T}$. The power consumption of such a system would be large, but the capital cost probably far less than that of a recirculating accelerator.

\subsection{Rotating and Superconducting Hybrid}

Pulsed magnets would be used up to the highest possible energy; say 0.5 $\mathrm{TeV}$. A sequence of two hybrid accelerators could then be used: one from $0.5-1 \mathrm{TeV}$, and the other from 1-2 TeV. Each would employ $8 \mathrm{~T}$ superconducting fixed magnets alternating now with pairs of counter-rotating permanent magnets [27]. Fields as high as $2 \mathrm{~T}$ should be possible in magnets of outside diameter less than $20 \mathrm{~cm}$.

If, for example, the superconducting magnets are 1.5 times the lengths of 
the two rotating magnets, then as the rotating magnets turn, the average field will swing from $\frac{(1.5 x 8-2-2)}{3.5}=2.3 \mathrm{~T}$ to $\frac{(1.5 x 8+2+2)}{3.5}=4.6$; varying by a factor of 2. For the final stage ( 1 to $2 \mathrm{TeV})$ : with acceleration in 20 turns, and a ring circumference of $20 \mathrm{~km}$, the acceleration time would be $1.3 \mathrm{msec}$, requiring a rotation rate of about $15,000 \mathrm{rpm}$. For the penultimate stage $(0.5$ to $1 \mathrm{TeV})$ : again with acceleration in 20 turns, and a ring circumference of $10 \mathrm{~km}$, the acceleration time would be $0.7 \mathrm{msec}$, requiring a rotation rate of about 30,000 $\mathrm{rpm}$. If 30,000 rpm is too high, 40 turn acceleration could be used in this stage and the rotation rate kept at 15,000 rpm. This should be practical, and the power consumption would be negligible. However, many technical questions remain to be answered.

\section{COLLIDER STORAGE RING}

After acceleration, the $\mu^{+}$and $\mu^{-}$bunches are injected into a separate storage ring. The highest possible average bending field is desirable, to maximize the number of revolutions before decay, and thus maximize the luminosity. Collisions would occur in one, or perhaps two, very low- $\beta^{*}$ interaction areas. Parameters of the ring were given earlier in Tb.1.

\subsection{Bending Magnet Design}

The magnet design is complicated by the fact that the $\mu$ 's decay within the rings $\left(\mu^{-} \rightarrow e^{-} \overline{\nu_{e}} \nu_{\mu}\right)$, producing electrons whose mean energy is approximately 0.35 that of the muons. These electrons travel toward the inside of the ring dipoles, radiating a fraction of their energy as synchrotron radiation towards the outside of the ring, and depositing the rest on the inside. The total average power deposited, in the ring, in the $4 \mathrm{TeV}$ machine is $13 \mathrm{MW}$, yet the maximum power that can reasonably be taken from the magnet coils at $4 \mathrm{~K}$ is only of the order of $40 \mathrm{KW}$. The power deposited could be reduced if the beams are kicked out of the ring prior to their their complete decay. Since the luminosity goes as the square of the number of muons, a significant power reduction can be obtained for a small luminosity loss. But still the power level is high. Two promising approaches are discussed below.

\subsubsection{Large Cosine-Theta Magnet}

The beam is surrounded by a thick warm shield, located inside a large aperture conventional cosine-theta magnet (see Fig.14). Fig.15 shows the attenuation of the heating produced as a function of the thickness of a warm tungsten liner [28]. If conventional superconductor is used, then the thicknesses required 


\begin{tabular}{|c|c|c|c|c|}
\hline & & $2 \mathrm{TeV}$ & $0.5 \mathrm{TeV}$ & Demo \\
\hline Unshielded Power & MW & 13 & 1.6 & .26 \\
\hline Liner inside rad & $\mathrm{cm}$ & 2 & 2 & 2 \\
\hline Liner thickness & $\mathrm{cm}$ & 6 & 4 & 2 \\
\hline Coil inside rad & $\mathrm{cm}$ & 9 & 7 & 5 \\
\hline Attenuation & & 400 & 80 & 12 \\
\hline Power leakage & KW & 32 & 20 & 20 \\
\hline Wall power for $4 K$ & MW & 26 & 16 & 16 \\
\hline
\end{tabular}

TABLE 6. Thickness of Shielding for Cos Theta Collider Magnets.

in the three cases would be as given in Tb.6. If high Tc superconductors could be used, then these thicknesses could probably be halved.

If this approach were taken, then the quadrupoles would best use warm iron poles placed as close to the beam as practical. The coils could be either superconducting or warm, as dictated by cost considerations. If an elliptical vacuum chamber were used, and the poles were at $1 \mathrm{~cm}$ radius, then gradients of $150 \mathrm{~T} / \mathrm{m}$ should be possible.

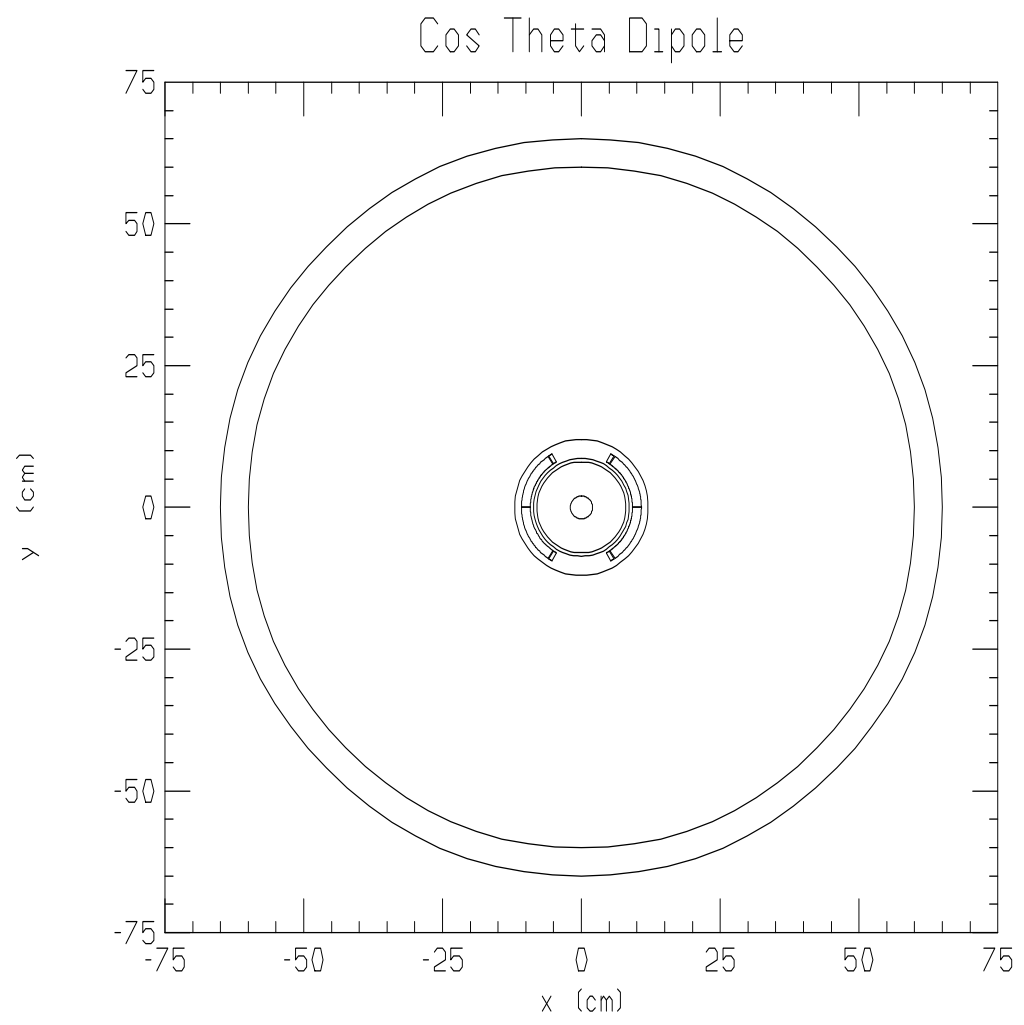

FIGURE 14. Cos Theta Arc Bending Magnet 


\subsection{2 ' $C$ ' Magnets}

An alternative is to use ' $\mathrm{C}$ ' magnets facing inward, with a broad vacuum pipe extending out of the gaps (see Fig.16). The decay electrons will spiral inward, out of the gap of the magnet, and can be absorbed in a separate warm dump. Some of the synchrotron radiation will still strike the inner wall of the vacuum chamber and a more limited dump is required there.

With $\mathrm{Nb}_{3} \mathrm{Sn}$ conductors, there appear no theoretical problems in achieving $10 \mathrm{~T}$ fields with very good field quality $\left(d B / B \leq 10^{-5}\right.$ for $\left.x \leq 1 \mathrm{~cm}\right)$. The problems would be in supporting the coils and maintaining the required position accuracy.

If this approach were chosen, then the quadrupoles could also be made as ' $\mathrm{C}$ ' magnets (see Fig.17). In such magnets there would be two pancake coils above and two below the vacuum chamber, with the current directions, in the two coils, opposite. This arrangement would generate a downward field to the left of the beam and an upward field to the right with a linear gradient, i.e. quadrupole field, in the center (see Fig.18) Again there appear no theoretical problems in defining coil blocks that achieve good field quality, and gradients of about $230 \mathrm{~T} / \mathrm{m}$ seem practical with $\mathrm{Nb}_{3} \mathrm{Sn}$ conductors. Again, the problems would be in supporting the coils and maintaining the required

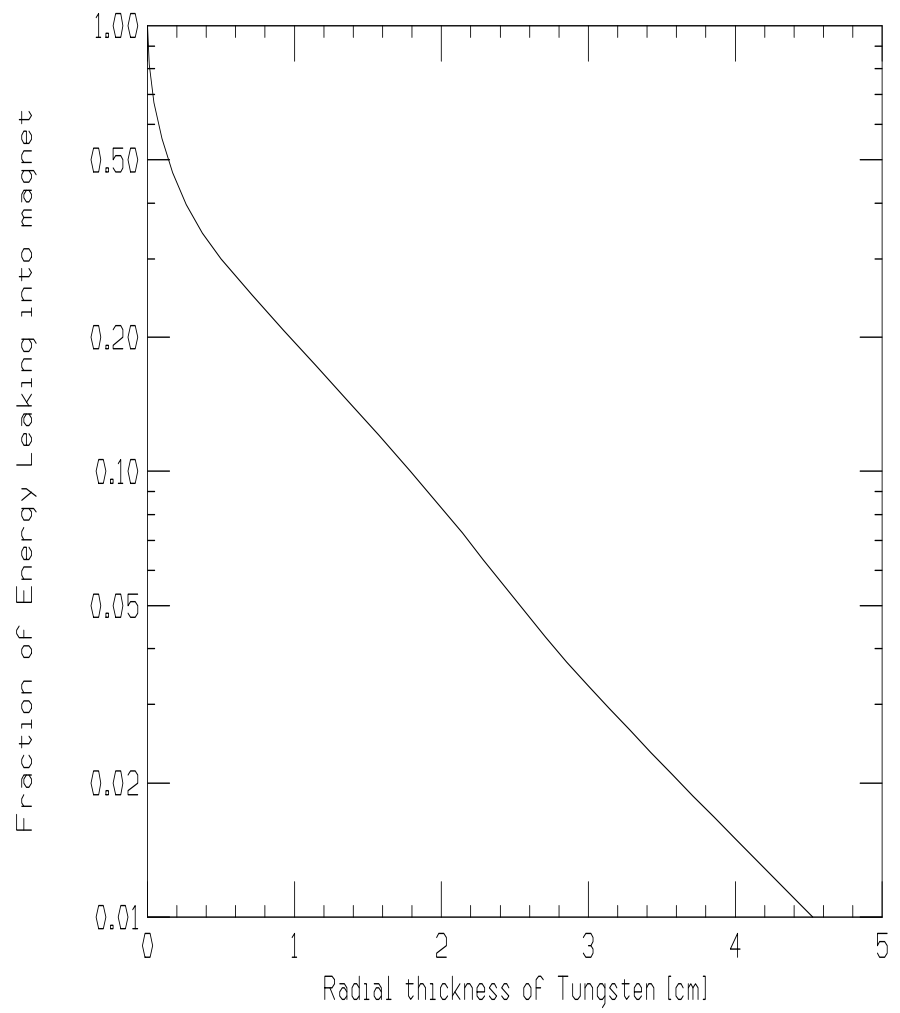

FIGURE 15. Energy attenuation vs the thickness of a tungsten liner. 
conductor position accuracy.

\subsubsection{Discussion}

The ' $\mathrm{C}$ ' magnet designs would require about $2 / 3$ of the superconductor needed for the cos theta magnets, but their overall size would not be much smaller. The simple pancake coils of the ' $\mathrm{C}$ ' magnets could be easier to wind than the cos theta type, but the support of the coils would be a new challenge. It is not yet known whether the thermal load would be greater or less in a ' $\mathrm{C}$ ' magnet design. Clearly, more study is needed to determine which approach is best.

\section{$5.2 \quad$ Lattice Design}

\subsubsection{Arcs}

In a conventional $2 \mathrm{TeV}$ superconducting ring the tune would be of the order of 200 and the momentum compaction $\alpha$ around $2 \times 10^{-3}$. In this case, in order to maintain a bunch with rms length $3 \mathrm{~mm}, 45 \mathrm{GeV}$ of S-band $\mathrm{rf}$

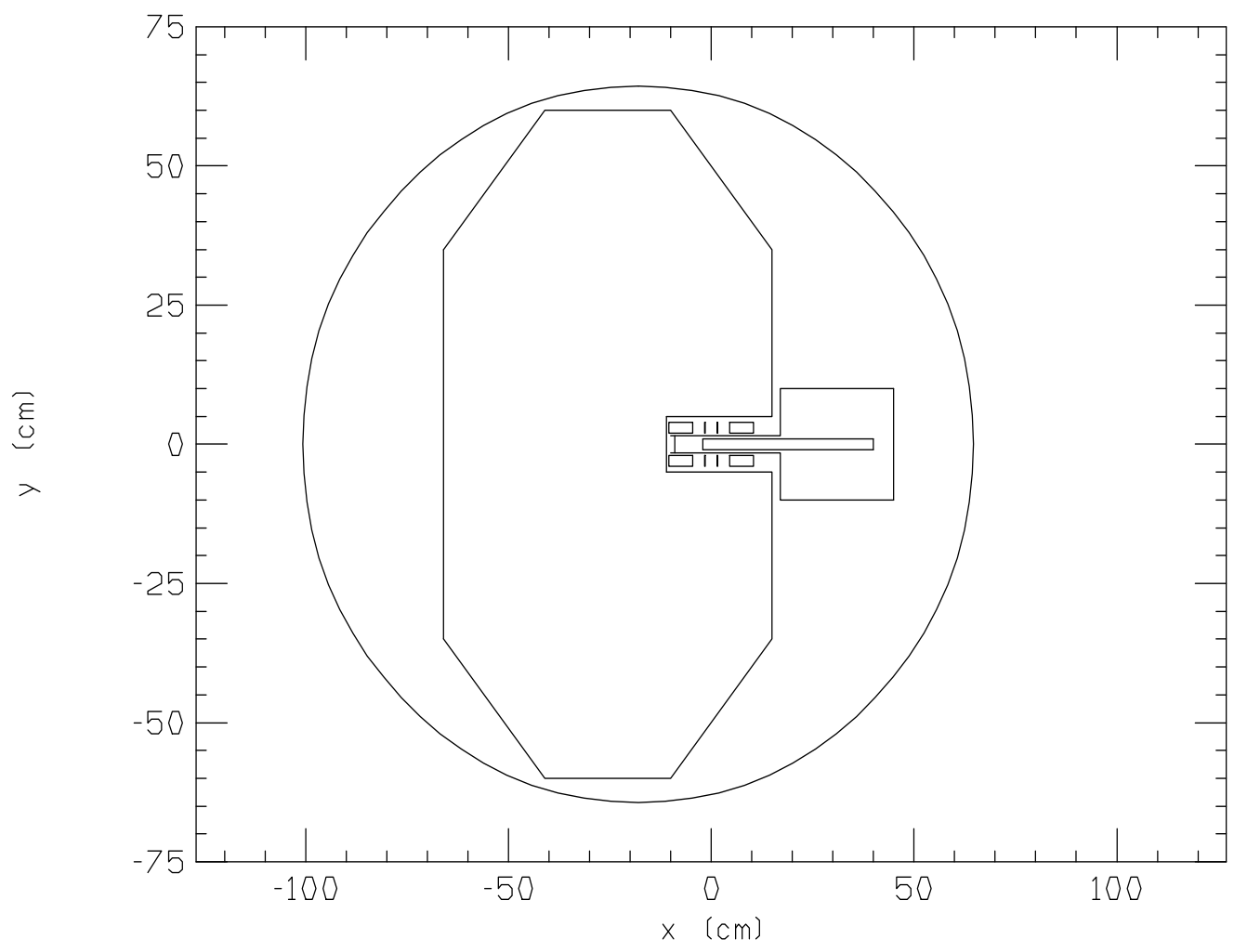

FIGURE 16. 'C' Arc Bending Magnet. 
would be required. This would be excessive. It is thus proposed to use an approximately isochronous lattice of the dispersion wave type [29]. Ideally one would like an $\alpha$ of the order of $10^{-7}$. In this case no $\mathrm{rf}$ would be needed to maintain the bunch and the machine would behave more like a linear beam transport. In practice it appears easy to set the zero'th order slip factor $\eta_{0}$ to zero, but if nothing is done, there is a relatively large first order slip factor $\eta_{1}$ yielding a maximum $\alpha$ of the order of $10^{-5}$. The use of sextupoles appears able to correct this $\eta_{1}$ yielding a maximum $\alpha$ of the order of $10^{-6}$. With octupoles it may be possible to correct $\eta_{2}$, but this remains to be seen.

It had been feared that amplitude dependent anisochronisity generated in the insertion would cause bunch growth in an otherwise purely isochronous design. It has, however, been pointed out [30] that if chromaticity is corrected in the ring, then amplitude dependent anisochronisity is automatically removed.

\subsubsection{Low $\beta$ Insertion}

In order to obtain the desired luminosity we require a very low beta at the intersection point: $\beta^{*}=3 \mathrm{~mm}$ for $4 \mathrm{TeV}, \beta^{*}=8 \mathrm{~mm}$ for the $.5 \mathrm{TeV}$ design. A possible final focusing quadruplet design is shown in Fig.19. The parameters of the quadrupoles for this quadruplet are given in Tb.7. The maximum fields

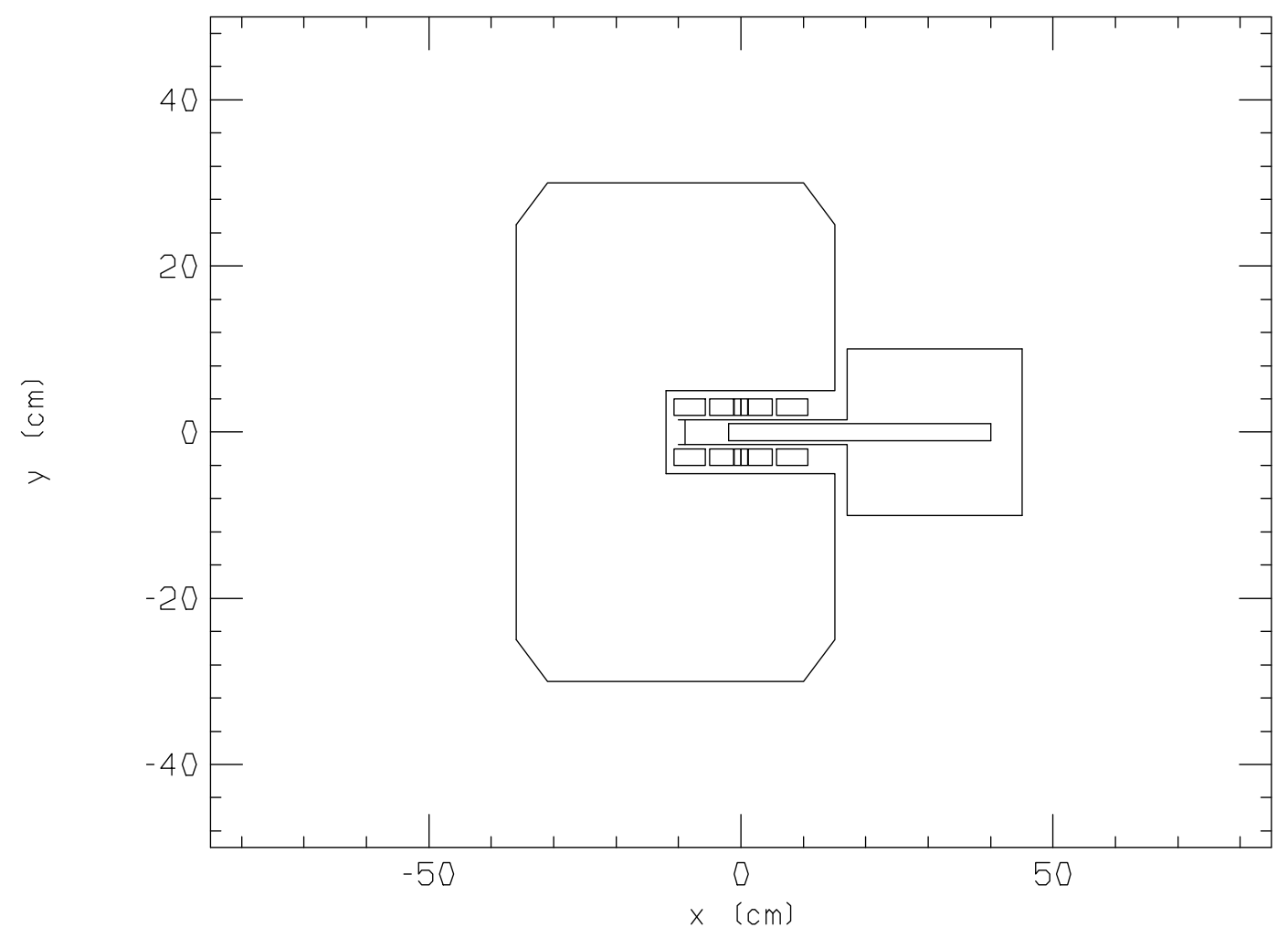

FIGURE 17. 'C' Arc Quadrupole. 
at 4 sigma have been assumed to be $6.4 \mathrm{~T}$. This would allow a radiation shield of the order of $5 \mathrm{~cm}$, while keeping the peak fields at the conductors less than $10 \mathrm{~T}$, which should be possible using $\mathrm{Nb}_{3} \mathrm{Sn}$ conductor.

With these elements, the maximum beta's in both $\mathrm{x}$ and $\mathrm{y}$ are of the order of $400 \mathrm{~km}$ in the $4 \mathrm{TeV}$ case, and $14 \mathrm{~km}$ in the $0.5 \mathrm{TeV}$ machine. The chromaticities $\left(1 / 4 \pi \int \beta d k\right)$ are approximately 6000 for the $4 \mathrm{TeV}$ case, and 600 for the $.5 \mathrm{TeV}$ machine. Such chromaticities are too large to correct within the rest of a conventional ring and therefore require local correction [31].

A preliminary model design [32] of local chromatic correction has been generated for the $4 \mathrm{TeV}$ case, and has been incorporated into a dispersion wave lattice [33]. Fig.20 shows the tune shift as a function of momentum. It is seen that this design has a momentum acceptance of $\pm 0.35 \%$. The second order amplitude dependent tune shifts shown in Fig.21 are less than 0.03 at one sigma ( 0.27 at 3 sigma in amplitude), which may be too large, even for only 1000 turns. In addition, this design used some bending fields that are unrealistic. It is expected that these limitations will soon be overcome, and that more sophisticated designs [34] should achieve a momentum acceptance of $\pm 0.6 \%$ for use with a clipped rms momentum spread of $0.2 \%$.

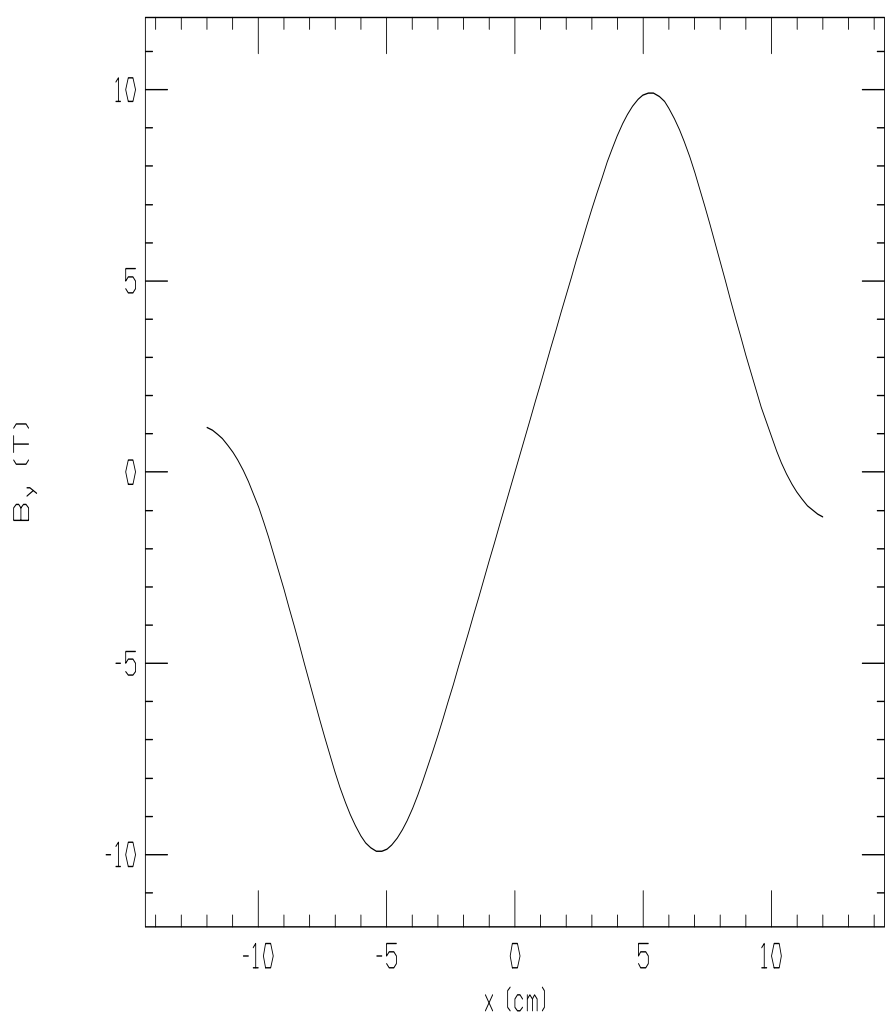

FIGURE 18. Vertical field as function of horizontal position in 'C' Quadrupole. 


\begin{tabular}{lccccc}
\hline & & \multicolumn{2}{c}{$4 \mathrm{MeV}$} & \multicolumn{2}{c}{$0.5 \mathrm{MeV}$} \\
\hline & field $(\mathrm{T})$ & $\mathrm{L}(\mathrm{m})$ & $\mathrm{R}(\mathrm{cm})$ & $\mathrm{L}(\mathrm{m})$ & $\mathrm{R}(\mathrm{cm})$ \\
\hline drift & & 6.5 & & 1.99 & \\
focus & 6 & 6.43 & 6 & 1.969 & 5.625 \\
drift & & 4.0 & & 1.2247 & \\
defocus & 6.4 & 13.144 & 12 & 4.025 & 11.25 \\
drift & & 4.0 & & 1.2247 & \\
focus & 6.4 & 11.458 & 12 & 3.508 & 11.25 \\
drift & & 4.0 & & 1.2247 & \\
defocus & 6.348 & 4.575 & 10 & 1.400 & 9.375 \\
drift & & 80 & & 24.48 & \\
\hline
\end{tabular}

TABLE 7. Final Focus Quadrupoles; L and $\mathrm{R}$ are the length and the radius respectively.

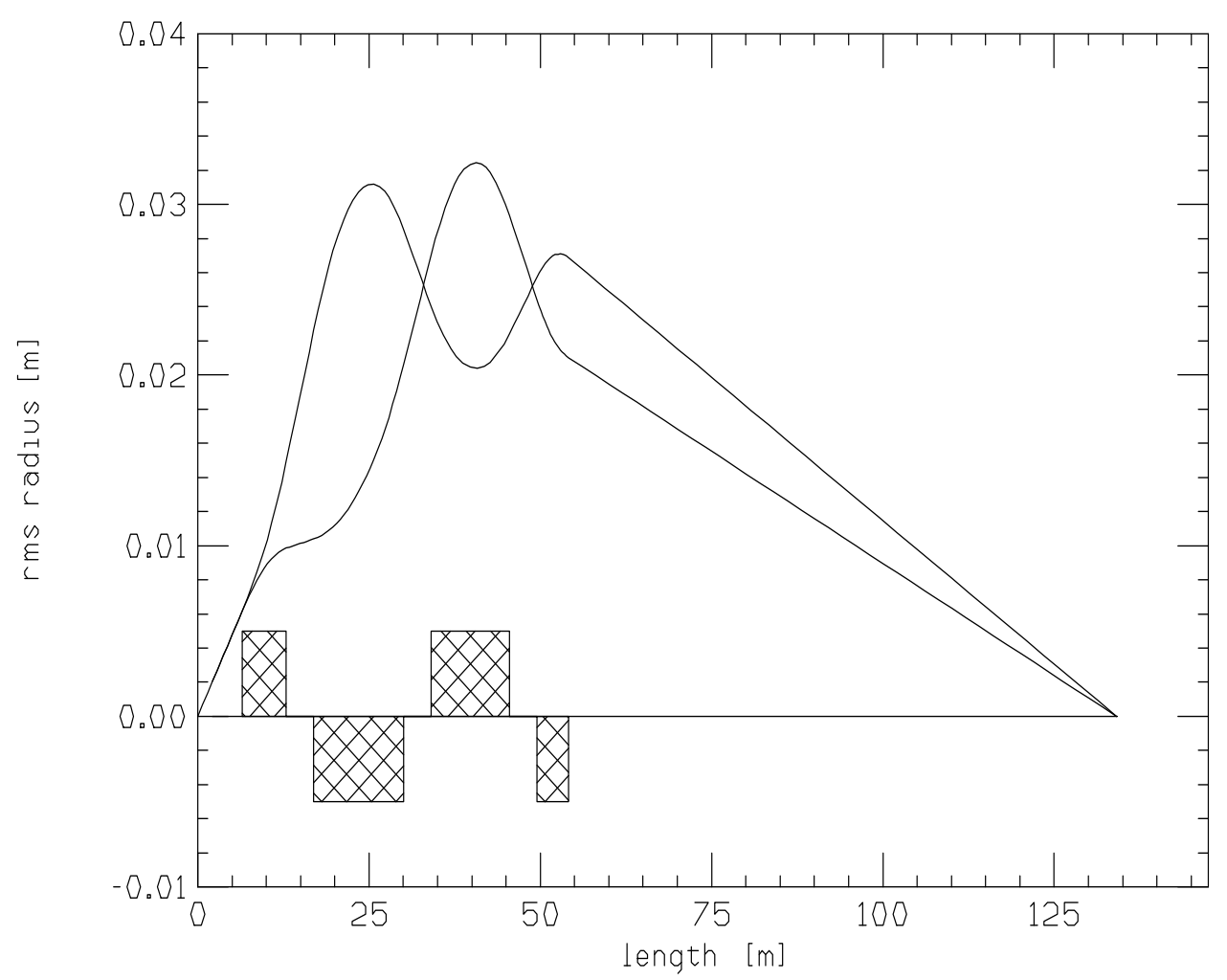

FIGURE 19. rms radius of the beam at the last four quadrupoles of the final focus.

\subsection{Instabilities}

Studies [35] of the resistive wall impedance instabilities indicate that the required muon bunches (eg for $2 \mathrm{TeV}: \sigma_{z}=3 \mathrm{~mm}, N_{\mu}=2 \times 10^{12}$ ) would be unstable in a conventional ring. In any case, the rf requirements to maintain such bunches would be excessive.

If one can obtain momentum-compaction factor $\alpha \leq 10^{-7}$, then the syn- 


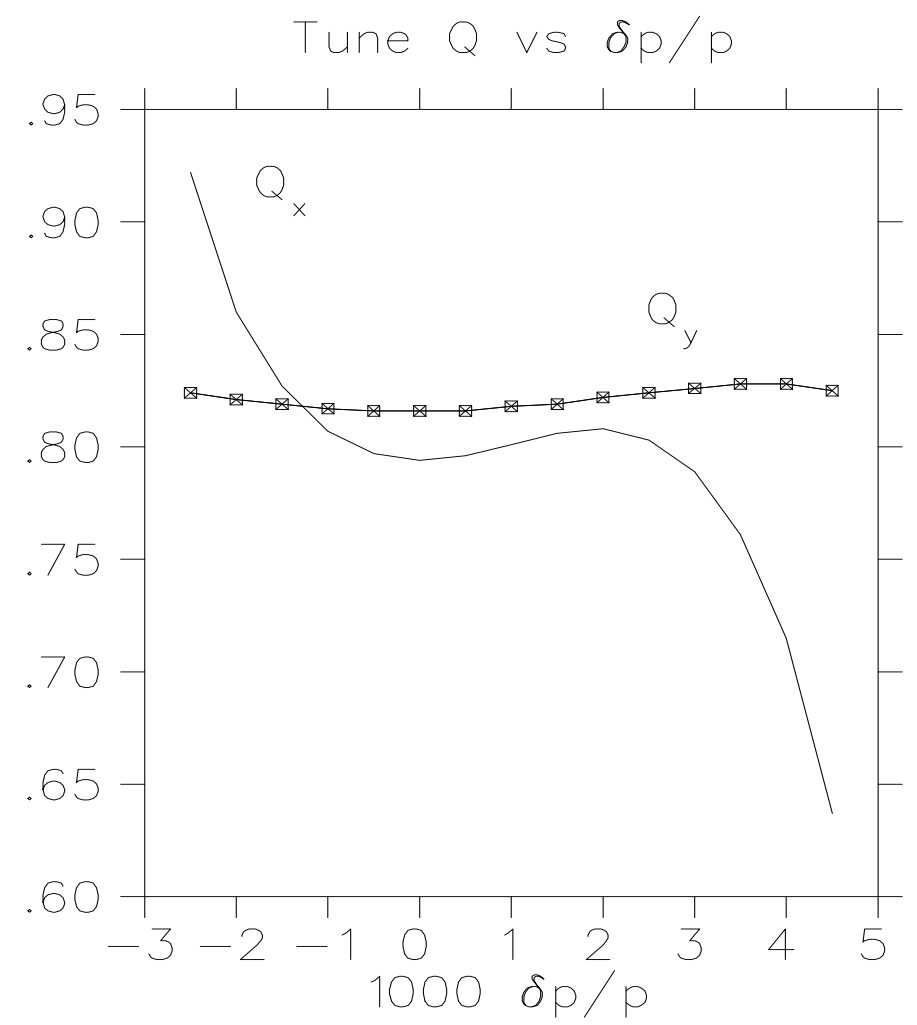

FIGURE 20. The tune shift as a function of $\Delta p / p$.

chrotron oscillation period is longer than the effective storage time, and the beam dynamics in the collider behave like that in a linear beam transport [36] [37]. In this case, beam breakup instabilities are the most important collective effects. Even with an aluminum beam pipe of radius $b=2.5 \mathrm{~cm}$, the resistive wall effect will cause the tail amplitude of the bunch to double in about 500 turns. For a broad-band impedance of $Q=1$ and $Z_{\|} / n=1 \mathrm{Ohm}$, the doubling time in the same beam pipe is only about 130 turns; which is clearly unacceptable. But both these instabilities can easily be stabilized using BNS [38] damping. For instance, to stabilize the resistive wall instability, the required tune spread, calculated [36] using the two particle model approximation, is (for $\mathrm{Al}$ pipe)

$$
\frac{\Delta \nu_{\beta}}{\nu_{\beta}}= \begin{cases}1.5810^{-4} & b=1.0 \mathrm{~cm} \\ 1.0710^{-5} & b=2.5 \mathrm{~cm} \\ 1.2610^{-6} & b=5.0 \mathrm{~cm}\end{cases}
$$

But this application of the BNS damping to a quasi-isochronous ring, where there are other head-tail instabilities due to the chromaticities $\xi$ and $\eta_{1}$, needs more careful study.

If it is not possible to obtain an $\alpha$ less than $10^{-7}$, then rf must be introduced and synchrotron oscillations will occur. The above instabilities are 


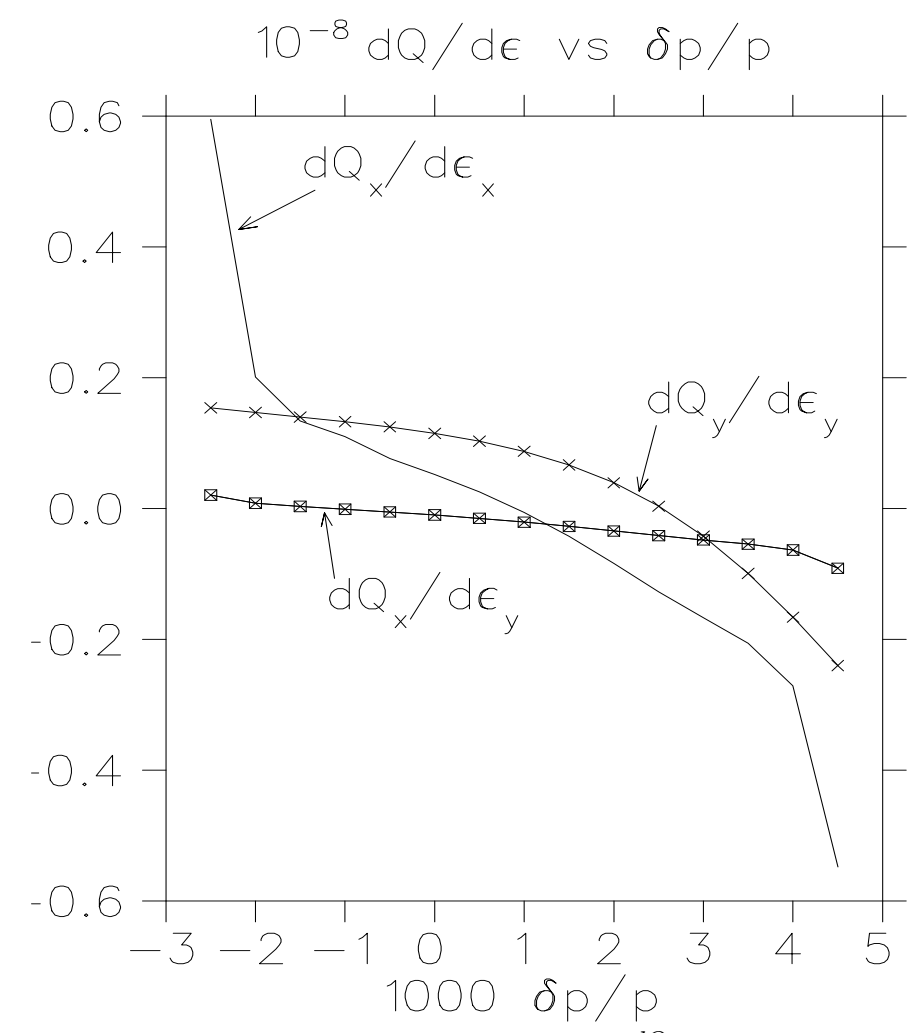

FIGURE 21. Amplitude dependent tune shift $\frac{d Q}{d \epsilon}$ as a functions of $\Delta p / p$.

then somewhat stabilized because of the interchanging of head and tail, but the impedance of the rf now adds to the problem and simple BNS damping is no longer possible.

For example, a momentum-compaction factor $|\alpha| \approx 1.5 \times 10^{-5}$ has been studied; rf of $\sim 1.5 \mathrm{GV}$ is needed which gives a synchrotron oscillation period of 150 turns. Three different impedance models: resonator, resistive wall, and a SLAC-like or a CEBAF-like rf accelerating structure have been used in the estimation for three sets of design parameters. The impedance of the ring is dominated by the rf cavities, and the microwave instability is well beyond threshold. Two approaches are being considered to control these instabilities: 1) BNS damping applied by rf quadrupoles as suggested by Chao [39]; and 2) applying an oscillating perturbation on the chromaticity [40].

When the ring is nearly isochronous, a longitudinal head-tail (LHT) instability may occur because the nonlinear slip factor $\eta_{1}$ becomes more important than the first order $\eta_{0}$ [3]. The growth time for the rf impedance when $\eta \simeq 10^{-5}$ is about $0.125 b \eta_{0} / \eta_{1} \mathrm{~s}$, where $b$ is the pipe radius in $\mathrm{cm}$. This would be longer than the storage time of $\sim 41 \mathrm{~ms}$ if $\eta_{1} \sim \eta_{0}$. However, if $\eta_{1} \sim \eta_{0} / \delta$, with $\delta \sim 10^{-3}$, then the growth time is about $0.125 b \mathrm{~ms}$, which is much shorter than the storage time. More study is needed. 


\section{COLLIDER PERFORMANCE}

\subsection{Luminosity vs Energy and Momentum Spread}

The bunch populations decay exponentially, yielding an integrated luminosity equal to its initial value multiplied by an effective number of turns $n_{\text {effective }}=150 \mathrm{~B}$, where $\mathrm{B}$ is the mean bending field in $\mathrm{T}$.

The luminosity is given by:

$$
\mathcal{L}=\frac{\mathrm{N}^{2} \mathrm{f} \mathrm{n} \mathrm{n}_{\mathrm{e}} \gamma}{4 \pi \beta^{*} \epsilon_{\mathrm{n}}} \mathrm{H}(\mathrm{A}, \mathrm{D})
$$

where $A=\sigma_{z} / \beta^{*}$,

$$
D=\frac{\sigma_{z} N}{\gamma \sigma_{t}^{2}} r_{e}\left(\frac{m_{e}}{m_{\mu}}\right)
$$

and the enhancement factor is

$$
H(A, D) \approx 1+D^{1 / 4}\left[\frac{D^{3}}{1+D^{3}}\right]\left\{\ln (\sqrt{D}+1)+2 \ln \left(\frac{0.8}{A}\right)\right\} .
$$

In our case $\mathrm{A}=1, \mathrm{D} \approx .5$ and $\mathrm{H}(\mathrm{A}, \mathrm{D}) \approx 1[41]$.

For a fixed collider lattice, operating at energies lower than the design value, the luminosity will fall as $\gamma^{3}$. One power comes from the $\gamma$ in Eq.5; a second comes from $n_{e}$, the effective number of turns, that is proportional to $\gamma$; the third factor comes from $\beta^{*}$, which must be increased proportional to $\gamma$ in order to keep the beam size constant within the focusing magnets. The bunch length $\sigma_{z}$ must also be increased proportional to $\gamma$ so that the required longitudinal phase space is not decreased; so $\mathrm{A}=\sigma_{z} / \beta^{*}$ remains constant.

In view of this rapid drop in luminosity with energy, it would be desirable to have separate collider rings at relatively close energy spacings: e.g. not more than factors of two apart.

If it is required to lower the energy spread $\Delta E / E$ at a fixed energy, then again the luminosity will fall. Given the same longitudinal phase space, the bunch length $\sigma_{z}$ must be increased. If the final focus is retuned to simultaneously increase $\beta^{*}$ to maintain the same value of $A$, then the luminosity will be exactly proportional to $\Delta E / E$. But if, instead, the $\beta^{*}$ is kept constant, and the parameter $\mathrm{A}$ allowed to increase, then the luminosity falls initially at a somewhat lower rate. The luminosity, for small $\Delta E / E$ is then approximately given by:

$$
\mathcal{L}=2 \mathcal{L}_{0} \frac{\Delta \mathrm{E} / \mathrm{E}}{(\Delta \mathrm{E} / \mathrm{E})_{0}} .
$$

There may, however, be beam-beam tune shift emittance growth problems in this case. 


\subsection{Detector Background}

There will be backgrounds from the decay of muons in the ring, from muon halo around the beam, and from the interactions themselves.

\subsubsection{Muon Decay Background}

A first Monte Carlo study [44] of the muon decay background was done with the MARS95 code [13], based on a preliminary insertion lattice. A tungsten shielding nose was introduced, extending to within $15 \mathrm{~cm}$ of the intersection point. It was found that:

- a large part of the electromagnetic background came from synchrotron radiation, from the bending magnets in the chromatic correction section.

- as many as 500 hits per $\mathrm{cm}^{2}$ were expected in a vertex detector, falling off to the order of 2 hits per $\mathrm{cm}^{2}$ in an outer tracker.

- there was considerable, very low energy, neutron background: of the order of 30,000 neutrons per $\mathrm{cm}^{2}$, giving, with an efficiency of $310^{-3}$, about 100 hits per $\mathrm{cm}^{2}$.

It was hoped that by improving the shielding these backgrounds could be substantially reduced.

A more recent study [45] of the electromagnetic component of the background has been done using the GEANT codes [46]. This study differed from the first in several ways:

- the shower electrons and photons were followed down to a lower energy (50 keV for electrons and $15 \mathrm{keV}$ for photons).

- the nose angle, i.e. the angle not seen by the detector, was increased from 9 to 20 degrees to reduce radial shower penetration.

- the nose design was modified (see Fig.22) so that: 1) The incoming electrons are collimated to $\pm 4 \times \sigma_{\theta_{0}}$ (where $\sigma_{\theta_{0}}$ is the rms divergence of the beam) by a cone leading down towards the vertex. 2) The detector could not see any surface directly illuminated by these initial electrons, whether seen in the forward or backward (albedo) directions. 3) The detector could not see any surface that is illuminated by secondary electrons if the secondary scattering angle is forward. 4) The minimum distance through the collimator from the detector to any primarily illuminated surface was more than $100 \mathrm{~mm}$, and from any secondarily illuminated surface, $30 \mathrm{~mm}$.

- it was assumed that a collimator placed at a focus $130 \mathrm{~m}$ from the intersection point would be able to effectively shield all synchrotron photons 

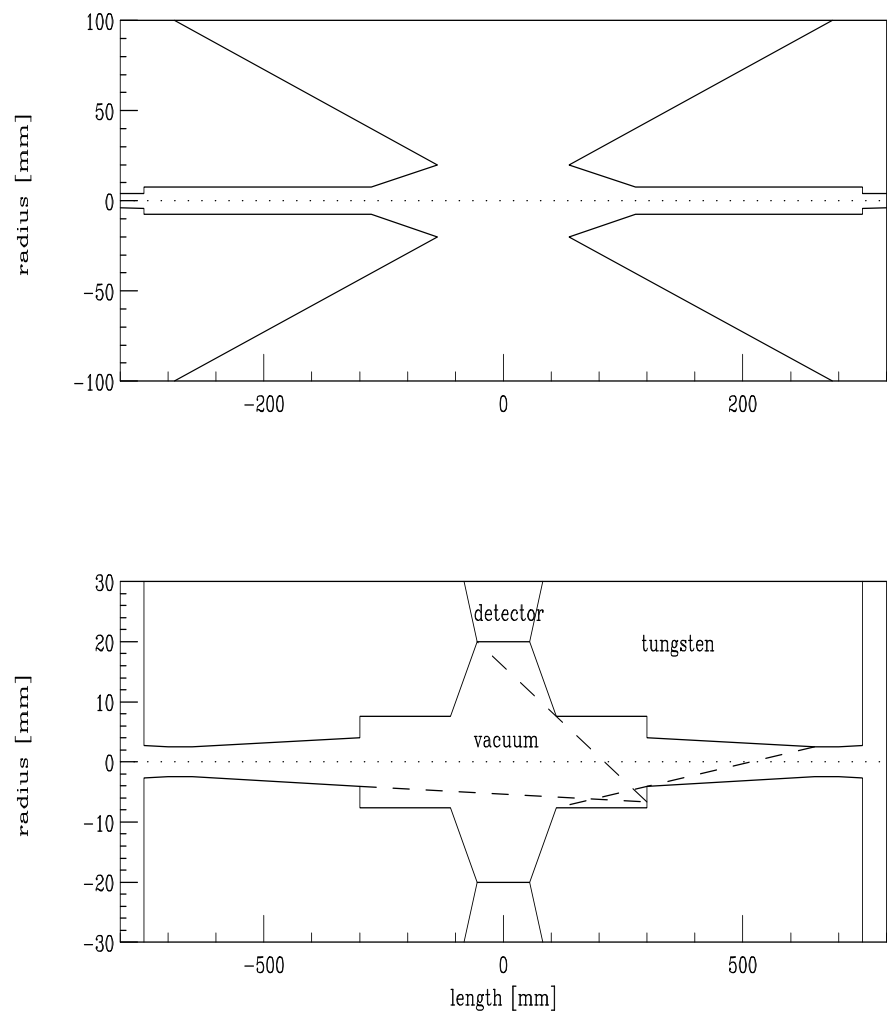

FIGURE 22. Schematic of the detector nose.

from the bending magnets beyond that point. The rms beam size at this focus is only $10 \mu \mathrm{m}$ so a very effective collimation should be possible (see Fig.19).

This study indicated that the dominant background was no longer from synchrotron photons, but from photons from $\mu$ decay electrons. The average momentum of these photons was only $1 \mathrm{MeV}$. Tb. 8 gives the total numbers of photons, the total number of hits, possible pixel sizes, and the hits per pixel, for a) a vertex detector placed at a $5 \mathrm{~cm}$ radius, and b) a gas detector placed at a $1 \mathrm{~m}$ radius. In all cases the numbers are given per bunch crossing. The sensitivities given here are for a silicon strip detector $(.3 \%)$ at the small radius, and a pad readout gas chamber (.1\%) at the larger radius. Both sensitivities could be reduced. Silicon strip detectors could be developed with less thickness, and a time projection chamber (TPC) could be used at the larger radii. The use of the TPC would be particularly advantageous because, not only is its density lower, but the small depositions of ionization from low energy photons and neutrons could not be mistaken for real tracks.

This study also found a relatively modest flux of muons from $\mu$ pair production in electromagnetic showers: about 50 such tracks pass through the detector per bunch crossing. 


\begin{tabular}{lcc}
\hline Detector & vertex & tracker \\
Radius & $5 \mathrm{~cm}$ & $1 \mathrm{~m}$ \\
\hline Number of photons & $5010^{6}$ & $1510^{6}$ \\
Number of hits & 150,000 & 15,000 \\
Detector Area & $863 \mathrm{~cm}^{2}$ & $34 \mathrm{~m}^{2}$ \\
Pixel size & $20 \times 20 \mu \mathrm{m} 1 \mathrm{~mm} \mathrm{x} 1 \mathrm{~cm}$ \\
Sensitivity & $0.3 \%$ & $0.1 \%$ \\
Occupancy & $.07 \%$ & $0.4 \%$ \\
\hline
\end{tabular}

TABLE 8. Detector Backgrounds from $\mu$ decay

The general conclusion of the two studies are not inconsistent as a cursory look may indicate. The background, though serious, is not impossible to overcome. Further reductions are expected as the shielding is optimized, and, as mentioned above, it should be possible to design detectors that are less sensitive to the neutrons and photons present.

\subsubsection{Muon Halo Background}

There would be a very serious background from the presence of even a very small halo of nearly full energy muons in the circulating beam [47]. The beam will need careful preparation before injection into the collider, and a collimation system will have to be designed to be located on the opposite side of the ring from the detector.

\subsubsection{Electron Pair Background}

In $e^{+} e^{-}$machines there is a significant problem from beamstrahlung photons (synchrotron radiation from beam particles in the coherent field of the oncoming bunch), and an additional problem from pair production by these photons.

With muons, there is negligible beamstrahlung, and thus negligible pair production from them. P. Chen [48] has further shown that beamstrahlung of electrons from the nearby decay of muons does not pose a problem.

There is, however, significant incoherent (i.e. $\mu^{+} \mu^{-} \rightarrow e^{+} e^{-}$) pair production in the $4 \mathrm{TeV}$ Collider case. The cross section is estimated to be $10 \mathrm{mb}$ [49], which would give rise to a background of $\approx 310^{4}$ electron pairs per bunch crossing. Approximately $90 \%$ of these, will be trapped inside the tungsten nose cone, but those with energy between 30 and $100 \mathrm{MeV}$ will enter the detector region.

There remains some question about the coherent pair production generated by the virtual photons interacting with the coherent electromagnetic fields 
of the entire oncoming bunch. A simple Weizsäcker-Williams calculation [50] yields a background that would consume the entire beam at a rate comparable with its decay. However, I. Ginzburg [51] and others have argued that the integration must be cut off due to the finite size of the final electrons. If this

is true, then the background becomes negligible. A more detailed study of this problem is now underway [52].

If the coherent pair production problem is confirmed, then there are two possible solutions:

1) one could design a two ring, four beam machine (a $\mu^{+}$and a $\mu^{-}$bunch coming from each side of the collision region, at the same time). In this case the coherent electromagnetic fields at the intersection are canceled and the pair production becomes negligible.

2) plasma could be introduced at the intersection point to cancel the beam electromagnetic fields [53].

\subsection{Polarization}

\subsubsection{Polarized Muon Production}

The generation of polarized muons has not yet received enough attention and the specifications and components described above have not been designed or optimized for polarization. Nevertheless, simple manipulations of parameters and/or the addition of simple components would allow some polarization with relatively modest loss of luminosity.

In the center of mass of a decaying pion, the outgoing muon is fully polarized (-1 for $\mu^{+}$and +1 for $\mu^{-}$). In the lab system the polarization depends [42] on the decay angle $\theta_{d}$ and initial pion energy. For pion kinetic energy larger than the pion mass, the dependence on pion energy becomes negligible and the polarization is given approximately by:

$$
P_{\mu^{-}} \approx \cos \theta_{d}+0.28\left(1-\cos ^{2} \theta_{d}\right)
$$

The average value of this is about 0.19 . At lower pion energies the polarization is higher, and has a value of the order of 0.5 at a kinetic energy of $10 \mathrm{MeV}$. If nothing is done, the polarization of the muons captured and phase rotated by the proposed system is approximately $20 \%$.

If higher polarization is required, some selection of muons from forward pion decays $\left(\cos \theta_{d} \rightarrow 1\right)$ is required. This could be done by selecting pions within a narrow energy range and then selecting only those muons with energy close to that of the selected pions. But such a procedure would collect a very small fraction of all possible muons and would yield a very small luminosity. Instead we wish, as in the unpolarized case, to capture pions over a wide energy range, allow them to decay, and to use rf to phase rotate the resulting distribution. 
Consider the distributions in velocity vs ct at the end of a decay channel. If the source bunch of protons is very short and if the pions were generated in the forward direction, then the pions, if they did not decay, would all be found on a single curved line. Muons from forward decays would have gained velocity and would lie above that line. Muons from backward decays would have lost velocity and would fall below the line. The real distribution would be diluted by the width of the proton bunch and the finite pion angles. In order to reduce the latter, it is found desirable to lower the solenoid field in the decay channel from 5 to 3 Tesla. When this is done one obtains the distribution shown in Fig.23, where the polarization $\mathrm{P}>\frac{1}{3}, \frac{-1}{3}<P<\frac{1}{3}$, and $\mathrm{P}<\frac{-1}{3}$ is marked by the symbols '+', '?' and '-' respectively.

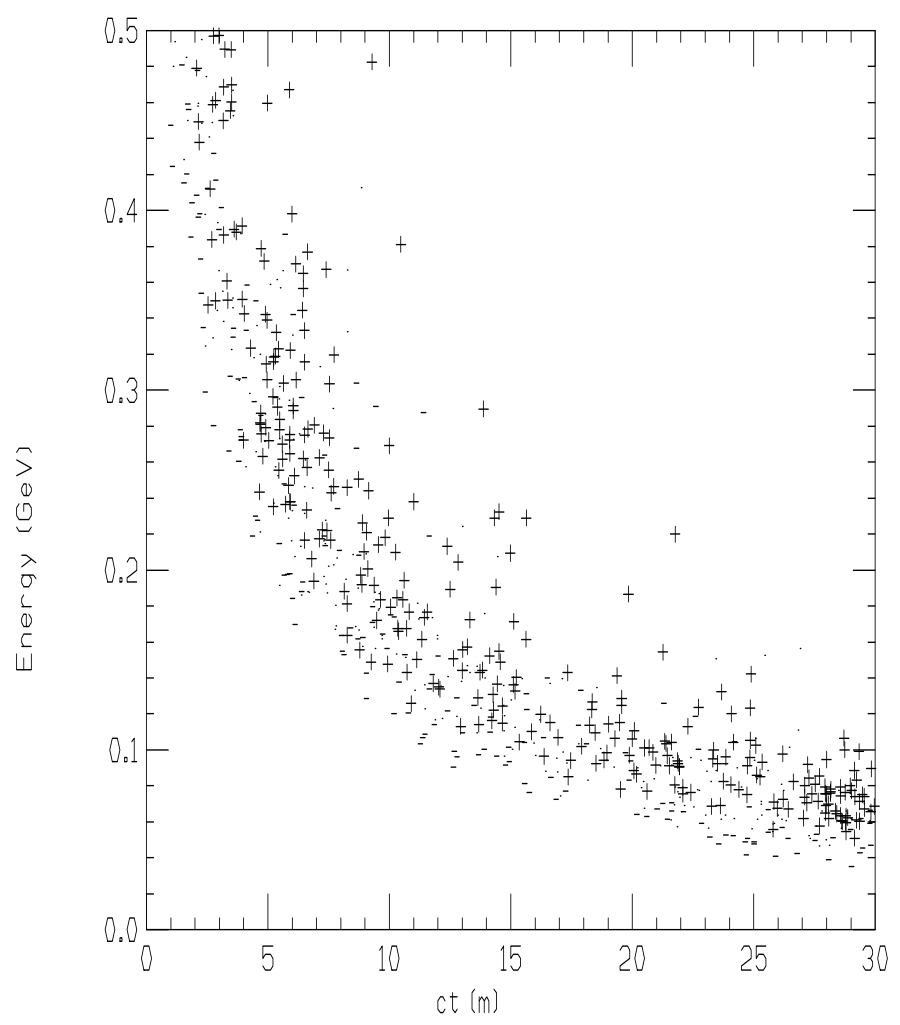

FIGURE 23. Energy vs ct of $\mu$ 's at end of decay channel ( no phase rotation).

After phase rotation with rf the correlation is preserved: see Fig.24 where as before the polarization $\mathrm{P}>\frac{1}{3}, \frac{-1}{3}<P<\frac{1}{3}$, and $\mathrm{P}<\frac{-1}{3}$ is marked by the symbols ' + ', '.' and '-' respectively.

If a selection is made on the minimum energy of the muons, then net polarization is obtained. The tighter the cut on energy, the higher the polarization, but the less the fraction $F_{\mu}$ of muons that are selected. Fig.25 gives the results of a Monte Carlo study.

The loss, about $30 \%$, from the use of the lower solenoid field, is included in the fractions $F_{\mu}$ plotted. 


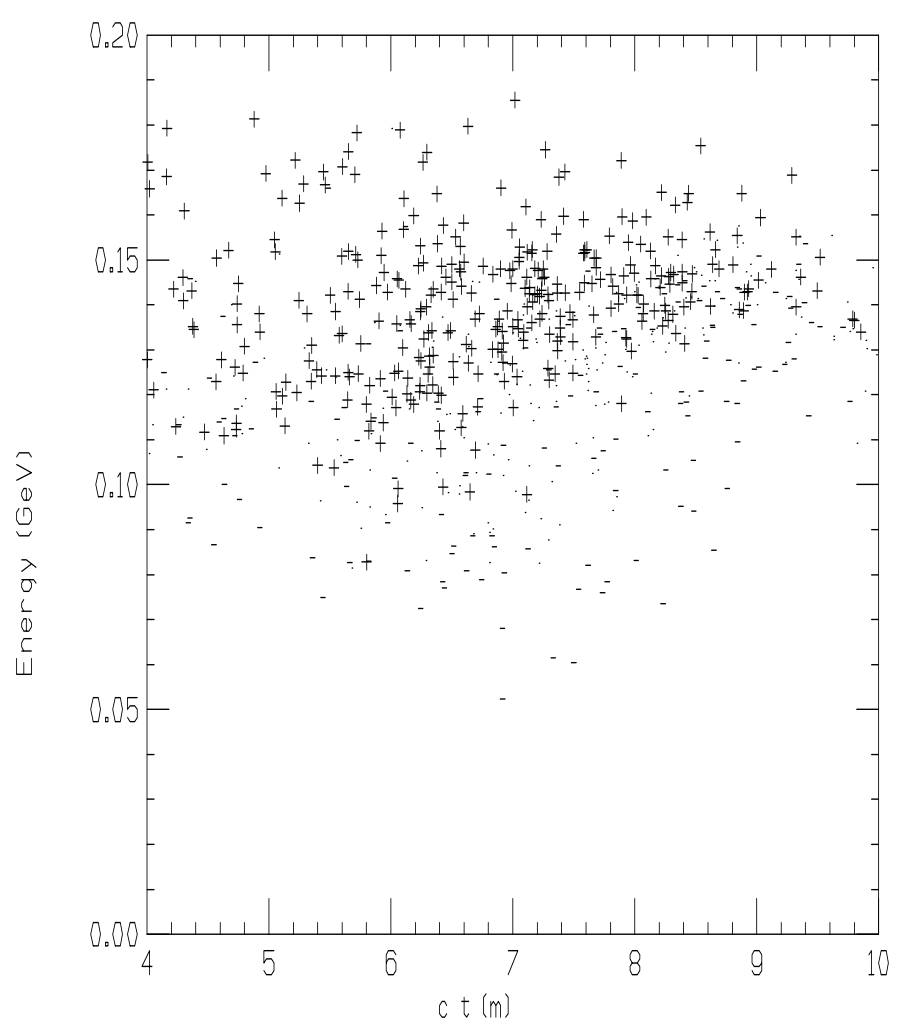

FIGURE 24. Energy vs ct of $\mu$ 's at end of decay channel with phase rotation.

\subsubsection{Polarization Preservation}

A recent paper [43] has discussed the preservation of muon polarization in some detail. During the ionization cooling process the muons lose energy in material and have a spin flip probability $\mathcal{P}$,

$$
\mathcal{P} \approx \int \frac{m_{e}}{m_{\mu}} \beta_{v}^{2} \frac{d E}{E}
$$

where $\beta_{v}$ is the muon velocity divided by $\mathrm{c}$, and $\mathrm{dE} / \mathrm{E}$ is the fractional loss of energy due to ionization loss. In our case the integrated energy loss is approximately $3 \mathrm{GeV}$ and the typical energy is $150 \mathrm{MeV}$, so the integrated spin flip probability is close to $10 \%$. The change in polarization $d P / P$ is twice the spin flip probability, so the reduction in polarization is approximately $20 \%$.

During circulation in any ring, the muon spins, if initially longitudinal, will precess by (g-2) $/ 2 \gamma$ turns per revolution in the ring; where $(\mathrm{g}-2) / 2$ is $1.16610^{-3}$. A given energy spread $d \gamma / \gamma$ will introduce variations in these precessions and cause dilution of the polarization. But if the particles remain in the ring for an exact integer number of synchrotron oscillations, then their individual average $\gamma$ 's will be the same and no dilution will occur. It appears 


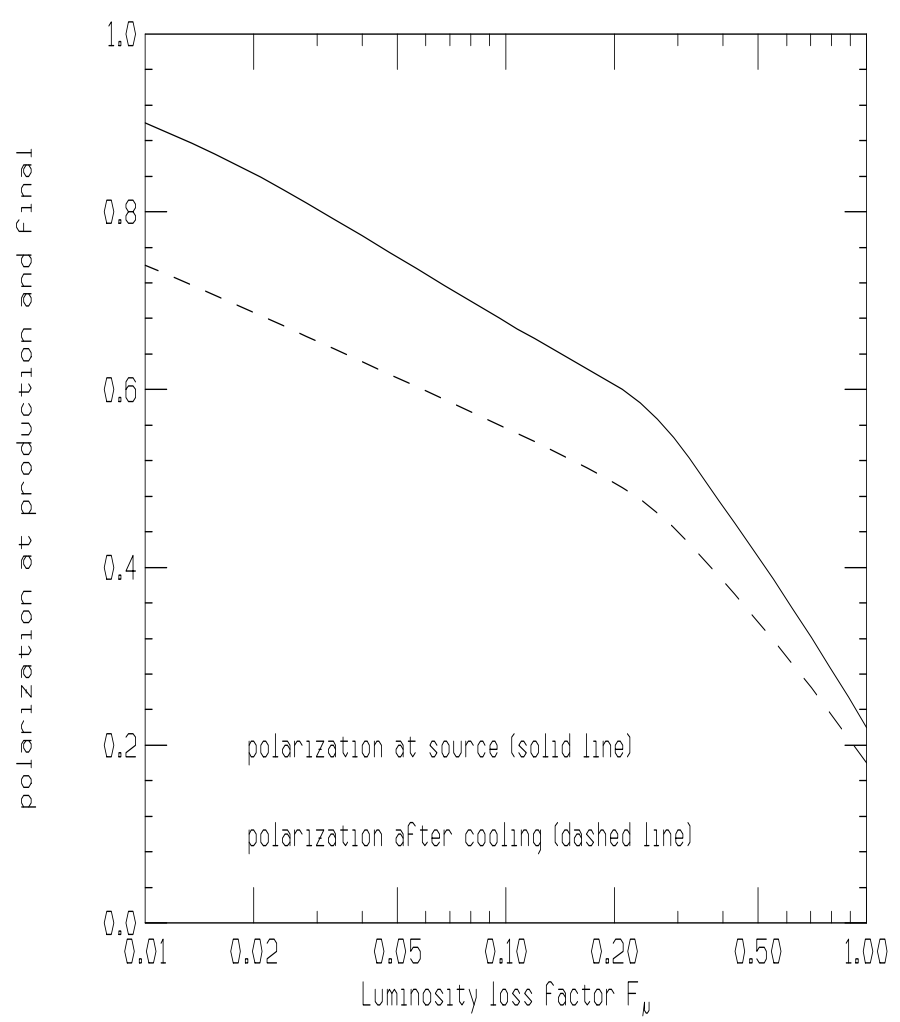

FIGURE 25. Polarization vs $F_{\mu}$ of $\mu$ 's accepted.

reasonable to use this 'synchrotron spin matching' [43] to avoid dilution during acceleration.

In the collider, however, the synchrotron frequency will probably be too slow to use 'synchrotron spin matching', so one of two methods must be used.

- Bending can be performed with the spin orientation in the vertical direction, and the spin rotated into the longitudinal direction only for the interaction region. The design of such spin rotators appears relatively straightforward. The example given in the above reference would only add $120 \mathrm{~m}$ of additional arc length, but no design has yet been incorporated into the lattice.

- The alternative is to install a $120 \mathrm{~m} 10 \mathrm{~T}$ solenoid (Siberian Snake) at a location exactly opposite to the intersection point. Such a solenoid reverses the sign of the horizontal polarization and generates a cancellation of the precession in the two halves of the ring.

Provision must also be made to allow changes in the relative spins of the two opposing bunches. This could be done, prior to acceleration, by switching one of the two beams into one or the other of two alternative injection lines. 


\subsubsection{Luminosity loss with polarization}

If both muon beams are polarized, then naturally the luminosity would drop as the square of the fraction $F_{\mu}$ of selected $\mu$ 's shown in Fig.25, but the loss need not be so great. In the unpolarized case of the $4 \mathrm{TeV}$ collider, there were two bunches of each sign. If the $F_{\mu}$ chosen for polarization is less than 0.5 , then this number of bunches could be reduced to one, without introducing excessive beam-beam tune shift. A factor of two in luminosity is then restored. If, for instance, the $F_{\mu}$ is taken as 0.5 for both signs, and the number of bunches is reduced to one of each sign, then the luminosity is reduced by a factor of only 0.5 and not 0.25 .

One also notes that the luminosity could be maintained at the full unpolarized value if the proton source intensity could be increased. Such an increase in proton source intensity in the unpolarized case would be impractical because of the resultant excessive high energy muon beam power, but this restriction does not apply if the increase is used to offset losses in generating polarization. If, for instance, the driver repetition rate were increased from 15 to $30 \mathrm{~Hz}$, the fractions $F_{\mu}$ set at 0.5 , and the number of bunches reduced to one, then the full luminosity of $10^{35}\left(\mathrm{~cm}^{-2} \mathrm{~s}^{-1}\right)$ would be maintained with polarization of both beams of $35 \%$.

The numbers given in this section are preliminary. Optimization of the systems may improve the polarizations obtained, but other dilution mechanisms may reduce them.

\section{CONCLUSION}

- Considerable progress has been made on a scenario for a $2+2 \mathrm{TeV}$, high luminosity collider. Much work remains to be done, but no obvious show stopper has yet been found.

- The two areas that could present serious problems are: 1) unforeseen losses during the 25 stages of cooling (a $3 \%$ loss per stage would be very serious); and 2) the excessive detector background from muon beam halo.

- Many technical components require development: a large high field solenoid for capture, low frequency rf linacs, multi-beam pulsed and/or rotating magnets for acceleration, warm bore shielding inside high field dipoles for the collider, muon collimators and background shields, etc. but:

- None of the required components may be described as exotic, and their specifications are not far beyond what has been demonstrated.

- If the components can be developed and the problems can be overcome, then a muon-muon collider should be a viable tool for the study of high energy phenomena, complementary to $e^{+} e^{-}$and hadron colliders. 


\section{ACKNOWLEDGMENTS}

We acknowledge important contributions from our colleagues, especially W. Barletta, A. Chao, J. Irwin, H. Padamsee, C. Pellegrini and A. Ruggiero.

This research was supported by the U.S. Department of Energy under Contract No. DE-ACO2-76-CH00016 and DE-AC03-76SF00515.

\section{REFERENCES}

1. E. A. Perevedentsev and A. N. Skrinsky, Proc. 12th Int. Conf. on High Energy Accelerators, F. T. Cole and R. Donaldson, Eds., (1983) 485; A. N. Skrinsky and V.V. Parkhomchuk, Sov. J. of Nucl. Physics 12, (1981) 3; Early Concepts for $\mu^{+} \mu^{-}$Colliders and High Energy $\mu$ Storage Rings, Physics Potential 86 Development of $\mu^{+} \mu^{-}$Colliders. $2^{\text {nd }}$ Workshop, Sausalito, CA, Ed. D. Cline, AIP Press, Woodbury, New York, (1995).

2. D. Neuffer, Colliding Muon Beams at $90 \mathrm{GeV}$, Fermilab Physics Note FN319 (1979), unpublished; D. Neuffer, Particle Accelerators, 14, (1983) 75; D. Neuffer, Proc. 12th Int. Conf. on High Energy Accelerators, F. T. Cole and R. Donaldson, Eds., 481 (1983).

3. Proceedings of the Mini-Workshop on $\mu^{+} \mu^{-}$Colliders: Particle Physics and Design, Napa CA, Nucl Inst. and Meth., A350 (1994) ; Proceedings of the Muon Collider Workshop, February 22, 1993, Los Alamos National Laboratory Report LA- UR-93-866 (1993) and Physics Potential 83 Development of $\mu^{+} \mu^{-}$ Colliders $2^{\text {nd }}$ Workshop, Sausalito, CA, Ed. D. Cline, AIP Press, Woodbury, New York, (1995).

4. Transparencies at the $2+2 \mathrm{TeV} \mu^{+} \mu^{-}$Collider Collaboration Meeting, Feb 68, 1995, BNL, compiled by Juan C. Gallardo; transparencies at the $2+2 \mathrm{TeV}$ $\mu^{+} \mu^{-}$Collider Collaboration Meeting, July 11-13, 1995, FERMILAB, compiled by Robert Noble; Proceedings of the 9th Advanced ICFA Beam Dynamics Workshop, Ed. J. C. Gallardo, AIP Press, to be published.

5. Overall Parameters and Construction Techniques Working Group report, Proceedings of the Fifth International Workshop on Next-Generation Linear Colliders, Oct 13-21, 1993, Slac-436, pp.428.

6. D. V. Neuffer and R. B. Palmer, Proc. European Particle Acc. Conf., London (1994); M. Tigner, in Advanced Accelerator Concepts, Port Jefferson, NY 1992, AIP Conf. Proc. 279, 1 (1993).

7. R. B. Palmer et al., Monte Carlo Simulations of Muon Production, Physics Potential $\&$ Development of $\mu^{+} \mu^{-}$Colliders $2^{\text {nd }}$ Workshop, Sausalito, CA, Ed. D. Cline, AIP Press, Woodbury, New York, pp. 108 (1995).

8. T. Roser, AGS Performance and Upgrades: A Possible Proton Driver for a Muon Collider, Proceedings of the 9th Advanced ICFA Beam Dynamics Workshop, Ed. J. C. Gallardo, AIP Press, to be published.

9. Y. Cho, et al., A 10-GeV, 5-MeV Proton Source for a Pulsed Spallation Source, Proc. of the 13th Meeting of the Int'l Collaboration on Advanced Neutron 
Sources, PSI Villigen, Oct. 11-14 (1995); Y. Cho, et al., A 10-GeV, 5-MeV Proton Source for a Muon-Muon Collider, Proceedings of the 9th Advanced ICFA Beam Dynamics Workshop, Ed. J. C. Gallardo, AIP Press, to be published.

10. F. Mills, et al., presentation at the 9th Advanced ICFA Beam Dynamics Workshop, unpublished; see also second reference in [4].

11. T. Roser and J. Norem, private communication.

12. D. Kahana, et al., Proceedings of Heavy Ion Physics at the AGS-HIPAGS '93, Ed. G. S. Stephans, S. G. Steadman and W. E. Kehoe (1993); D. Kahana and Y. Torun, Analysis of Pion Production Data from E-802 at $14.6 \mathrm{GeV} / \mathrm{c}$ using ARC, BNL Report \# 61983 (1995).

13. N. V. Mokhov, The MARS Code System User's Guide, version 13(95), Fermilab-FN-628 (1995).

14. J. Ranft, DPMJET Code System (1995).

15. N. Mokhov, R. Noble and A. Van Ginneken, Target and Collection Optimization for Muon Colliders, Proceedings of the 9th Advanced ICFA Beam Dynamics Workshop, Ed. J. C. Gallardo, AIP Press, to be published.

16. R. Weggel, private communication; Physics Today, pp. 21-22, Dec. (1994).

17. R. Chehab, J. Math. Phys. 5, (1978) 19.

18. F. Chen, Introduction to Plasma Physics, Plenum, New York, pp. 23-26 (9174); T. Tajima, Computational Plasma Physics: With Applications to Fusion and Astrophysics, Addison-Wesley Publishing Co., New York, pp. 281-282 (1989).

19. A. A. Mikhailichenko and M. S. Zolotorev, Phys. Rev. Lett. 71, (1993) 4146; M. S. Zolotorev and A. A. Zholents, SLAC-PUB-6476 (1994).

20. A. Hershcovitch, Brookhaven National Report AGS/AD/Tech. Note No. 413 (1995).

21. Z. Huang, P. Chen and R. Ruth, SLAC-PUB-6745, Proc. Workshop on Advanced Accelerator Concepts, Lake Geneva, WI , June (1994); P. Sandler, A. Bogacz and D. Cline, Muon Cooling and Acceleration Experiment Using Muon Sources at Triumf, Physics Potential \& Development of $\mu^{+} \mu^{-}$Colliders $2^{\text {nd }}$ Workshop, Sausalito, CA, Ed. D. Cline, AIP Press, Woodbury, New York, pp. 146 (1995).

22. Initial speculations on ionization cooling have been variously attributed to $\mathrm{G}$. O’Neill and/or G. Budker see D. Neuffer in [2]; D. Neuffer, in Advanced Accelerator Concepts, AIP Conf. Proc. 156, 201 (1987); see also [1].

23. U. Fano, Ann. Rev. Nucl. Sci. 13, 1 (1963).

24. G. Silvestrov, Proceedings of the Muon Collider Workshop, February 22, 1993, Los Alamos National Laboratory Report LA-UR-93-866 (1993); B. Bayanov, J. Petrov, G. Silvestrov, J. MacLachlan, and G. Nicholls, Nucl. Inst. and Meth. 190, (1981) 9; Colin D. Johnson, Hyperfine Interactions, 44 (1988) 21; M. D. Church and J. P. Marriner, Annu. Rev. Nucl. Sci. 43 (1993) 253.

25. G. Silvestrov, Lithium Lenses for Muon Colliders, Proceedings of the 9th Advanced ICFA Beam Dynamics Workshop, Ed. J. C. Gallardo, AIP Press, to be published.

26. D. Neuffer, Acceleration to Collisions for the $\mu^{+} \mu^{-}$Collider, Proceedings of 
the 9th Advanced ICFA Beam Dynamics Workshop, Ed. J. C. Gallardo, AIP Press, to be published.

27. D. Summers, presentation at the 9th Advanced ICFA Beam Dynamics Workshop, unpublished.

28. I. Stumer, presentation at the BNL-LBL-FNAL Collaboration Meeting, Feb 1996, BNL, unpublished.

29. S.Y. Lee, K.-Y. Ng and D. Trbojevic, FNAL Report FN595 (1992); Phys. Rev. E48, (1993) 3040; D. Trbojevic, et al., Design of the Muon Collider Isochronous Storage Ring Lattice, Micro-Bunches Workshop, BNL Oct. (1995), to be published.

30. K. Oide, private communication.

31. K. L. Brown and J. Spencer, SLAC-PUB-2678 (1981) presented at the Particle Accelerator Conf., Washington, (1981) and K.L. Brown, SLAC-PUB-4811 (1988), Proc. Capri Workshop, June 1988 and J.J. Murray, K. L. Brown and T.H. Fieguth, Particle Accelerator Conf., Washington, 1987; Bruce Dunham and Olivier Napoly, FFADA, Final Focus. Automatic Design and Analysis, CERN Report CLIC Note 222, (1994); Olivier Napoly, it CLIC Final Focus System: Upgraded Version with Increased Bandwidth and Error Analysis, CERN Report CLIC Note 227, (1994).

32. J. C. Gallardo and R. B. Palmer, Final Focus System for a Muon Collider: A Test Model, BNL \#, CAP 138-MUON-96R (1996), this proceedings.

33. A. Garren, et al., Design of the Muon Collider Lattice: Present Status, this proceedings.

34. K. Oide, SLAC-PUB-4953 (1989); J. Irwin, SLAC-PUB-6197 and LBL-33276, Particle Accelerator Conf.,Washington, DC, May (1993); R. Brinkmann, Optimization of a Final Focus System for Large Momentum Bandwidth, DESY-M90/14 (1990).

35. M. Syphers, private communication; K.-Y. Ng, Beam Stability Issues in a Quasi-Isochronous Muon Collider, Proceedings of the 9th Advanced ICFA Beam Dynamics Workshop, Ed. J. C. Gallardo, AIP Press, to be published.

36. K.Y. Ng, Beam Stability Issues in a Quasi-Isochronous Muon Collider, Proceedings of the 9th Advanced ICFA Beam Dynamics Workshop, Ed. J. C. Gallardo, AIP Press, to be published.

37. W.-H. Cheng, A.M. Sessler, and J.S. Wurtele, Studies of Collective Instabilities, in Muon Collider Rings, Proceedings of the 9th Advanced ICFA Beam Dynamics Workshop, Ed. J. C. Gallardo, AIP Press, to be published.

38. V. Balakin, A. Novokhatski and V. Smirnov, Proc. 12th Int. Conf. on High Energy Accel., Batavia, IL, 1983, ed. F.T. Cole, Batavia: Fermi Natl. Accel. Lab. (1983), p. 119.

39. A. Chao, Physics of Collective Beam Instabilities in High Energy Accelerators, John Wiley \& Sons, Inc, New York (1993).

40. W.-H. Cheng, private communication.

41. P. Chen and K. Yokoya, Phys. Rev. D38 987 (1988); P. Chen., SLAC-PUB4823 (1987); Proc. Part. Accel. School, Batavia, IL, 1987; AIP Conf. Proc. 184: 633 (1987). 
42. K. Assamagan, et al., Phys Lett. B335, 231 (1994); E. P. Wigner, Ann. Math. 40, 194 (1939) and Rev. Mod. Phys., 29, 255 (1957).

43. B. Norum and R. Rossmanith, Polarized Beams in a Muon Collider, this proceedings.

44. G. W. Foster and N. V. Mokhov, Backgrounds and Detector Performance at 2 + $2 \mathrm{TeV} \mu^{+} \mu^{-}$Collider, Physics Potential $\&$ Development of $\mu^{+} \mu^{-}$Colliders $2^{\text {nd }}$ Workshop, Sausalito, CA, Ed. D. Cline, AIP Press, Woodbury, New York, pp. 178 (1995).

45. I. Stumer, private communication and presentation at the BNL-LBL-FNAL Collaboration Meeting, Feb. 1996, unpublished.

46. Geant Manual, Cern Program Library V. 3.21, Geneva, Switzerland, 1993.

47. N. V. Mokhov and S. I. Striganov, Simulation of Background in Detectors and Energy Diposition in Superconducting Magnets at $\mu^{+} \mu^{-}$Colliders, FermilabConf-96/011, Proceedings of the 9th Advanced ICFA Beam Dynamics Workshop, Ed. J. C. Gallardo, AIP Press, to be published.

48. P. Chen, presentation at the 9th Advanced ICFA Beam Dynamics Workshop and this conference, unpublished.

49. L. D. Landau and E. M. Lifshitz, Phys. Zs. Sowjetunion 6, 244 (1934); V. M. Budnev, I. F. Ginzburg, G. V. Medelin and V. G. Serbo, Phys Rep., 15C, 181 (1975).

50. P. Chen, presentation at the 9th Advanced ICFA Beam Dynamics Workshop and this conference, unpublished.

51. I. Ginzburg, private communication and this proceedings.

52. P. Chen and N. Kroll, private communication.

53. G. V. Stupakov and P. Chen, Plasma Suppression of Beam-Beam Interaction in Circular Colliders, SLAC Report: SLAC-PUB-95-7084 (1995) 


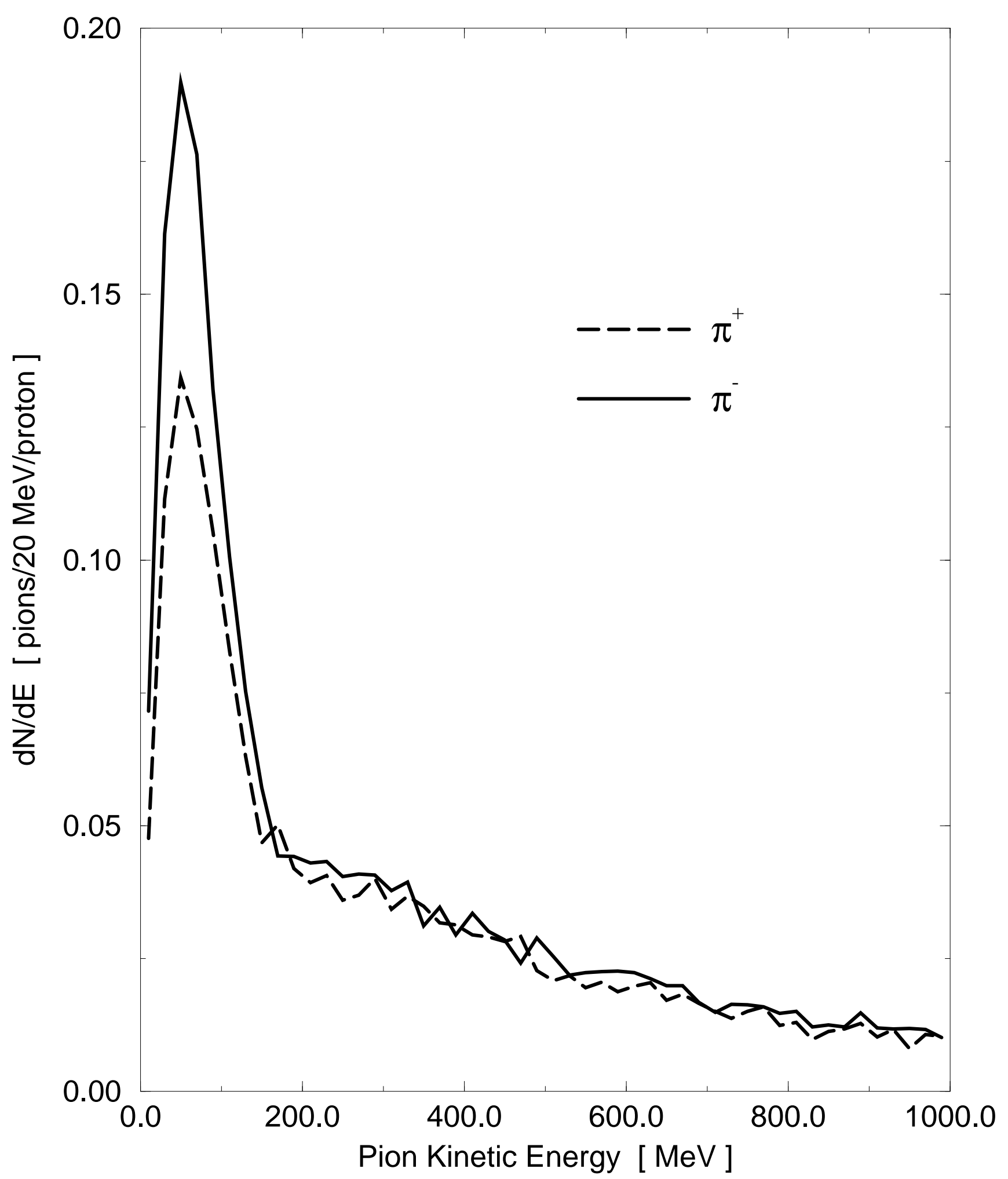

